\title{
Mapping the irradiance field of a single tree: quantifying vegetation induced adjacency effects
}

\author{
Daniel Kükenbrink, Andreas Hueni, Member, IEEE, Fabian D. Schneider \\ Alexander Damm, Jean-Philippe Gastellu-Etchegorry, \\ Michael E. Schaepman, Senior Member, IEEE, and Felix Morsdorf
}

\begin{abstract}
Imaging spectroscopy is frequently used to assess traits and functioning of vegetated ecosystems. Applied reflectance and radiance based approaches critically rely on accurate estimates of surface irradiance. Accurate retrievals of surface irradiance are, however, non- trivial and often error prone, thus causing inaccurate estimates of vegetation information. We analyse the irradiance field surrounding an isolated tree using the three-dimensional radiative transfer model DART in high spatial $(25 \mathrm{~cm})$ and spectral $(1 \mathrm{~nm}, 350-2500 \mathrm{~nm})$ resolution. We validate modeled irradiance with in situ measurements and quantify the impact of erroneous surface irradiance estimates on the retrieval of vegetation indices. We observe irradiance gradients in cast shadows of $<560 \%$ in the blue spectral range, while this gradient decreases with increasing wavelength and becomes negligible in the near infrared. Further, we quantify a vegetation induced decrease in irradiance of $<6 \%$ in the visible spectral region and an increase of $<7 \%$ in the near infrared outside the cast shadow. Commonly employed vegetation indices are also affected by such brightening or darkening effects. Outside the cast shadow, indices sensitive to the relative content of chlorophyll and carotenoids show an overestimation of $<14 \%$. The Photochemical Reflectance Index (PRI) shows an underestimation of $<5 \%$. Our study provides first quantitative insight in high spatial and spectral resolution, on the impact of vegetation on its surrounding irradiance field. Findings highlight important implications for vegetation assessments and provide the fundamental base to advance retrievals of vegetation traits and functioning from imaging spectroscopy data.
\end{abstract}

Index Terms

Atmospheric Correction, Cast Shadow, Imaging Spectroscopy, Irradiance Modeling, Radiative Transfer

This document is the accepted manuscript version of the following article:

Kükenbrink, D., Hueni, A., Schneider, F. D., Damm, A., Gastel1u-Etchegorry, J. P., Schaepman, M. E., \& Morsdorf, F. (2019). Mapping the irradiance field of a single tree: quantifying vegetationinduced adjacency effects. IEEE Transactions on Geoscience and Remote sensing, 57(7), 4994-5011. https://doi .org/10.1109/TGRS.2019.2895211

The research leading to these results has received funding from the European Union's 7th Framework Programme (FP7/2014 - 2018) under EUFAR2 contract n 312609. The contributions of Andreas Hueni, Fabian D. Schneider, Felix Morsdorf, and Michael E. Schaepman are supported by the University of Zurich Research Priority Program on 'Global Change and Biodiversity'. Contributions of Andreas Hueni are also supported by MetEOC-3 project, which has received funding from the EMPIR programme co-financed by the participating states and from the European Unions Horizon 2020 research and innovation programme. (Corresponding author: Daniel Kükenbrink)

Daniel Kükenbrink is with the Remote Sensing Laboratories, Department of Geography, University of Zürich, Winterthurerstrasse 190, CH-8057 Zürich, Switzerland (e-mail: daniel.kuekenbrink@geo.uzh.ch).

Andreas Hueni, Alexander Damm, Michael E. Schaepman and Felix Morsdorf are with the Remote Sensing Laboratories, Department of Geography, University of Zürich, 8057 Zürich, Switzerland (e-mail: ahueni@geo.uzh.ch, alexander.damm@geo.uzh.ch, michael.schaepman@geo.uzh.ch, felix.morsdorf@geo.uzh.ch)

Alexander Damm is further with the Department of Surface Waters - Research and Management, Eawag, Swiss Federal Institute of Aquatic Science and Technology, $\mathrm{CH}-8600$ Dübendorf, Switzerland.

Fabian D. Schneider is with the NASA Jet Propulsion Laboratory, California Institute of Technology, Pasadena, CA 91011, USA (e-mail: fabian.schneider@jpl.nasa.gov)

Jean-Philippe Gastellu-Etchegorry is with the Centre d'Etudes Spatiales de la BIOsphere (CESBIO) - Toulouse University (UPS, CNES, CNRS, IRD), 31401 Toulouse cedex 9, France (e-mail: jean-philippe.gastellu-etchegorry@cesbio.cnes.fr) 


\section{Mapping the irradiance field of a single tree: quantifying vegetation induced adjacency effects}

\section{INTRODUCTION}

$\mathbf{I}$ MAGING spectroscopy is frequently used for vegetation canopy assessments. Major focus lies on the retrieval of physiological, biochemical and structural traits and on monitoring of spatio-temporal variations in vegetation functioning, health and status [1]-[3]. The ever increasing spatial, temporal and spectral resolution of sensors further allow the retrieval and monitoring of increasingly complex vegetation information such as functional diversity [4], or signals related to plant photosynthesis (e.g. sun-induced chlorophyll fluorescence) [5]. Vegetation functioning can be analyzed by either measuring subtle changes of leaf reflected radiance or emitted fluorescence radiance by exploiting narrow atmospheric absorption features. For both approaches, accurate estimates of surface irradiance are crucial to retrieve either top-of-canopy (ToC) reflectance and related vegetation information or fluorescence. However, with increasing canopy complexity such as in forest ecosystems, accurate irradiance retrieval becomes a challenge. Particularly shadowing effects substantially influence the retrieval of ToC reflectance [6]. Multiple scattering within vegetation canopy can further lead to subtle changes in the irradiance field causing a brightening or darkening of the pixels' apparent reflectance [7]. In order to properly describe the complex irradiance fields in these environments, one can no longer assume uniformly flat Earth surfaces or use coarse digital elevation models (DEM) which do not resolve small scale height variabilities [7]-[9]. There are already multiple approaches published trying to minimize the impact of varying illumination effects. Asner et al. [10] or Malenovský et al. [11], for example, propose to only consider sunlit pixels. Adler-Golden et al. [12] suggested the use of matched filtering of reflectance data to detect and correct shadows. More sophisticated approaches employ auxiliary data to approximate irradiance variation caused by small scale canopy height variations: Friman et al. [13] used a digital object model derived from LiDAR data to better represent the canopy surface. However, approaches employing an elevation model to represent the canopy surface often assume the surface to be opaque, which is a simplifying assumption that is often violated, especially in forest canopies. More recent approaches try to overcome these issues by using complex three-dimensional radiative transfer models [7], [14] such as the Discrete Anisotropic Radiative Transfer Model DART [15], [16].

Even though the problem of irradiance variabilities in surface reflectance retrievals has been discussed frequently and multiple approaches already exist trying to compensate such illumination effects, few studies actually quantified the effect of vegetation canopy on the surrounding irradiance field in a spectrally and spatially resolved manner. In fact, results of Fawcett et al. [7] demonstrate that even with best efforts (i.e. using a combination of DART RT modeling and high resolution digital object models), artifacts in retrieved $\mathrm{ToC}$ reflectance data are present due to the complexity of irradiance fields and multiple scattering in complex vegetation canopies. However, light scattering mechanisms of single leaves and agricultural crops have already been analysed. Huete [17] demonstrated how multiple scattered light influences the spectral response of surrounding soils, shadows and vegetation in a crop canopy at different solar angles. Roberts et al. and Roberts [18], [19] analysed adjacency effects caused by a single synthetic leaf positioned above backgrounds of different reflectance characteristics using a simplified radiative transfer model at multiple wavelengths in the visible and near infrared. In a more recent publication, Stuckens et al. [20] analysed the impact of commonly used assumptions in radiative transfer models (e.g. the averaging of optical properties of randomly distributed leaves in a canopy and the representation of leaves as Lambertian scatterers) on simulated light scattering mechanisms for a citrus orchard. The work in [20] further led to multiple studies where the impact of background soil on the retrieved spectral signature of citrus orchards was analyzed and a model for reducing such background effects was implemented [21], [22]. Focusing more on cast shadows, Lynch [23] analyzed and quantified the subtle changes in irradiance inside a shadow 
cast by an artificial object on a white surface with a commercial RGB camera, giving a more detailed insight into the completely non-binary shadow phenomenon. However, the three wavebands used in this study (red, green and blue) only cover a very small part of the electromagnetic spectrum interesting for vegetation studies. Focusing on light scattering in forest ecosystems, Hilker et al. [24], [25] analyzed the effects of mutual shading of tree crowns on the prediction of photosynthetic light use efficiency in a coastal Douglas-fir forest. They mitigated the problem of modeling the translucent forest canopy by introducing a weighting, or transparency factor on the hillshade-derived shadow mask. However, also here, the variance in canopy lighting was only analyzed in a panchromatic manner, not accounting for different shadowing and brightening effects at changing wavelengths induced by neighboring trees. Takala and Mõttus [26] analyzed the spatial variation of the canopy photochemical reflectance index (PRI) with shadow fraction caused by leaf-level irradiation conditions. Even though they were able to analyze the irradiance conditions at different wavelengths, they were not able to directly measure the irradiance at the analyzed leaves but used the irradiance conditions of neighboring roads to derive leaf-level irradiance. This is most probably highly influenced by multiple scattering and neighborhood effects, making the transfer to leaf-level irradiance difficult. In fact, the importance of diffuse sky radiation for the retrieval of canopy PRI [5] and the subsequent calculation of light use efficiency [27] was recently documented.

In this study we model the irradiance field around a single isolated tree using the DART radiative transfer model and quantify the irradiance variability caused by the 3D object. The modeled irradiance values are validated with measured irradiance values around the tree using field spectroradiometers. Finally, we evaluate the impact of erroneous irradiance estimates on the retrieval of reflectance and vegetation information

\section{STUDY SITE AND MATERIALS}

\section{A. Study site}

For this experiment, a single isolated tree was selected located on top of a drumlin $23 \mathrm{~km}$ southeast of Zurich, Switzerland $\left(47^{\circ} 16^{\prime} 31.333^{\prime \prime} \mathrm{N} 8^{\circ} 48^{\prime} 46.870^{\prime \prime} \mathrm{E}\right)$. The Linden tree (Tilia cordata.) is $11.8 \mathrm{~m}$ high with a crown diameter of $12 \mathrm{~m}$. The crown base height is at $1.5 \mathrm{~m}$ and the tree shows an average Leaf Area Index (LAI) of $8.4 \mathrm{~m}^{2} \mathrm{~m}^{-2}$ estimated with the approach described in Section III-A1. The tree is surrounded by agriculturally used grassland that had been trimmed prior to the measurements. All measurements were performed on the $6^{\text {th }}$ of July 2017, just a few days after the northern hemisphere solar maximum. The sky was near-cloudless with a few temporary contrails and high cirrus clouds present and a visibility of up to $23 \mathrm{~km}$.

\section{METHODS}

\section{A. DART Parameterization}

The radiative transfer model used in this study to simulate the irradiance field around the tree was the Discrete Anisotropic Radiative Transfer Model DART (v5.6.0) [16]. DART simulates three-dimensional heterogeneous landscapes in three operating modes: flux-tracking, LiDAR, and Monte Carlo. Generally, a DART scene is defined by a voxel grid with a predefined size $(0.25 \mathrm{~m}$ in this study). It can simulate any scene element, including vegetation such as grass or tree crowns, with triangles. Additionally, complex three-dimensional objects represented as a triangular mesh with predefined optical properties can be imported. Here, vegetation is simulated as turbid medium, where each voxel can be parameterized by volume density, leaf angular distribution, and optical properties. A DART voxel can include vegetation turbid media as well as triangles with an arbitrary size, independent of the voxel size. In ray tracing, two types of radiation interactions are simulated: volume interaction within turbid voxels [28], and surface interaction on triangles [29]. Further details on the DART model can be found in [15], [16].

In this study, the flux tracking mode was used with sun and atmosphere as the only radiation sources. The approach to parameterize the DART model is closely following the one described in [30], where at-sensor 
TABLE I

TLS DATA ACQUISITION SPECIFICATIONS.

\begin{tabular}{lr}
\hline TLS Parameter & TLS specifications \\
\hline Acquisition dates & 6th of July 2017 \\
TLS Sensor & Riegl VZ-1000 \\
Laser wavelength [nm] & $1550 \mathrm{~nm}$ \\
Beam divergence [mrad] & 0.3 \\
Beam width when leaving instrument [mm] & 7 \\
Laser pulse repetition rate PRR [kHz] & 150 \\
Max. measurement range [m] & $950 \mathrm{~m}$ \\
Angular resolution [deg] & 0.02 \\
Accuracy [mm] & 8 \\
Precision [mm] & 5 \\
\hline
\end{tabular}

radiances for airborne imaging spectrometer data were simulated and compared to actual measurements of the Airborne Prism Experiment (APEX) Sensor [2]. Optical properties of the tree crown and the surrounding surfaces were defined based on measurements described in Section III-A3. The geometry of the tree crown as well as the vegetation density for each voxel was defined as described in Section III-A1. The leaf angle distribution was defined as spherical, based on leaf angle measurements conducted on a tilia cordata in Kew Gardens, London by Jan Pisek from the Tartu Observatory, Estonia (unpublished data). Additionally, the stem was parameterized following the approach described in Section III-A2. The optical property of the stem was defined according to the default bark optical property found in the DART optical properties database. The topography of the surface was extracted from drone images as described in Section III-A4. To simulate the atmosphere, DART can be used with standard gas and aerosol models as contained in the MODTRAN model [31]. We used the mid-latitude summer gas model and the rural aerosol model with a visibility of $23 \mathrm{~km}$. For each measured irradiance transect (see Section III-B) a new DART simulation with modified solar angles (solar azimuth and elevation angle) according to the observed solar angles at the beginning of each transect were simulated. A total of 1873 wavelengths with $1 \mathrm{~nm}$ spectral resolution were simulated for each transect covering the spectral range of $350-2500 \mathrm{~nm}$. Wavelengths associated with very low signal to noise ratios due to absorption bands have been excluded. The output of the DART model is a three-dimensional radiative budget with the amount of energy (in W $\mathrm{m}^{-2} \mathrm{~nm}^{-1}$ ) irradiant, absorbed and scattered at each voxel. The radiative budget can be further decomposed into direct, diffuse and coupled irradiance (irradiance after coupling with the atmosphere). In this study, we distinguish between bottom of atmosphere (BoA) and top of canopy (ToC) irradiance. BoA irradiance is defined as the irradiance of a homogeneously illuminated flat surface uninfluenced by geometric scattering effects caused by surrounding 3D objects (e.g. shadowing effects, reduction of sky view factor etc.). ToC irradiance is the actual surface irradiance at each location including the geometric scattering effects. When calculating reflectance factors we distinguish between apparent ToC reflectance based on the BoA irradiance and true ToC reflectance based on the actual ToC irradiance.

1) Vegetation density estimation: Vegetation density for each turbid medium voxel was estimated using terrestrial laser scanning (TLS) measurements acquired on the same day as the irradiance measurements. The TLS instrument was operated at a wavelength of $1550 \mathrm{~nm}$ and a pulse repetition frequency of 150 $\mathrm{kHz}$ and a maximum range of $950 \mathrm{~m}$ (Riegl VZ-1000, Riegl, Austria). A detailed description of the TLS data acquisition is given in Table I. A scan pattern of $0.02^{\circ}$ spacing between pulses and a field of view of $100^{\circ}$ by $90^{\circ}$ in vertical and horizontal directions was used. A total of four scans approximately 20 - $30 \mathrm{~m}$ away from the tree trunk were acquired. The chosen scan pattern minimized occlusion effects and enabled a detailed representation of the tree [32], [33]. Cylindrical reflective targets were placed on and around the tree as tie-points for the subsequent scan position co-registration. The co-registration as well as the filtering of the point cloud was performed in Riegl's RiscanPro Software package (RiscanPro v2.0.2 r7440, Riegl, Austria). The TLS point cloud was further visually co-registered to the drone point cloud (see Section III-A4). 
The vegetation density values per voxel were estimated using the AMAPvox Software package (version 1.0.1 r3410ffbe) developed by "botAnique Modélisation de l'Architecture des Plantes et des végétations" AMAP [34]. The AMAPvox model is tracing all laser pulses through a predefined voxel grid and calculates for each voxel the local transmittance computed from the ratio between exiting and entering energy normalized by the mean optical path length [34]. As the model cannot distinguish between leaf and woody material, the output of each voxel is therefore defined as the Plant Area Density (PAD) per voxel.

2) Stem Model Extraction: While the turbid medium assumption of DART is valid for the tree crown, it does not hold for the tree trunk. We therefore excluded voxels of the tree trunk from the estimated vegetation density grid and modeled the tree trunk based on the TLS point cloud. We therefore extracted all laser returns reflected from the tree trunk including the first major branches of the tree and fitted a mesh around it using a screened poisson reconstruction [35] implemented in the open-source 3D mesh processing software MeshLab (Version 2016.12) [36]. The extracted stem object can be imported into DART as a wavefront object file and is then treated as an opaque lambertian object that is not penetrable by solar radiation.

3) Optical Properties Measurements: Leaf optical properties (LOPs) were acquired using one of the field spectroradiometers described in Section III-B, but with an added leaf-clip. The fore-optic allows us to calculate leaf reflectance and transmittance by using a white and a black reference panel with known reflectance and following the procedure described in [37]:

$$
\begin{aligned}
\rho_{t} & =\frac{\rho_{t, \text { low }}^{*} \times \rho_{B, \text { high }}-\rho_{t, \text { high }}^{*} \times \rho_{B, \text { low }}}{\rho_{B, \text { high }}-\rho_{B, \text { low }}} \\
\tau^{2} & =\frac{\left[\rho_{t, \text { high }}-\rho_{t}\right] \times\left[1-\rho_{t} \times \rho_{B, \text { high }}\right]}{\rho_{B, \text { high }}}
\end{aligned}
$$

where $\rho_{t}$ and $\tau$ are the extracted reflectance and transmittance of the leaf, whereas $\rho_{t, l o w}^{*}$ and $\rho_{t, h i g h}^{*}$ are the measured reflectance of the leaf with the white and the black reference panel as background respectively and $\rho_{B, \text { high }}, \rho_{B, \text { low }}$ are the measured relectance of the white and black reference panel without the leaf. The instrument was set to reflectance mode to acquire the LOPs. A total of 20 randomly selected leaves were sampled, 10 from the lower crown part and 10 from higher up the crown. For the DART parameterization, the crown was divided into an upper and lower half (height threshold set to half of TLS measured crown length) and the average LOP retrieved from the respective halves were assigned.

Radiance measurements from the ground surrounding the tree were also acquired with one of the field spectroradiometers without the use of a fore-optic. Four ground measurements were acquired located to the North-West, South-West, South-East, and North-East by averaging the measured radiance of a 1 $\mathrm{m}^{2}$ patch. Reflectance values for these ground measurements were calculated by taking white reference measurements from spectralon panels before and after the ground measurements of each location.

4) Terrain extraction: The aerial ortho-mosaic of the study site was generated using a commercial DJI Inspire 1 drone with the X5 gimbal. A total of 98 images were acquired with an average flying altitude of $45.2 \mathrm{~m}$ above ground and with an along track overlap of 90\%, while across track overlap was $85 \%$. The matching of the geotagged images was done with AGISOFT Photoscan professional, resulting in a ground pixel resolution of $1.58 \mathrm{~cm}$ and a point density of 250 points $/ \mathrm{m}^{2}$. The re-projection error (relative error) was estimated to be 1.51 pixel, while the absolute error of the whole ortho-mosaic was estimated to be $0.84 \mathrm{~m}$.

\section{B. Irradiance Measurements}

Irradiance measurements were performed with two ASD FieldSpec Pro spectroradiometers (Analytical Spectral Devices, FieldSpec Pro) measuring the reflected radiance from a white reference spectralon, with nearly $100 \%$ lambertian scattering characteristics [38]. One spectroradiometer was positioned on a sunlit patch over $30 \mathrm{~m}$ away from the tree to assure no influence of the tree on the measured irradiance. The spectralon was mounted on a tripod roughly one meter above ground and was leveled to be horizontal. The 


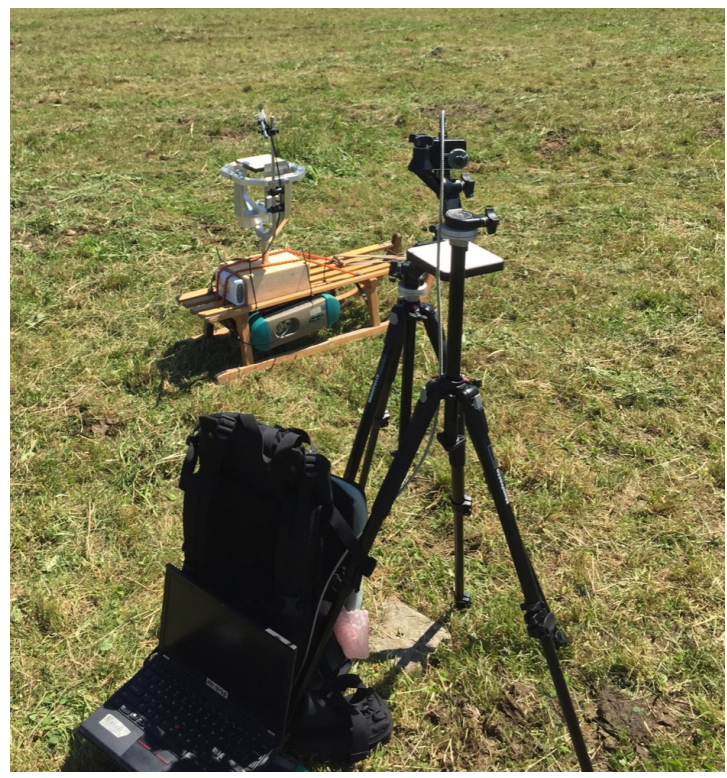

(a)

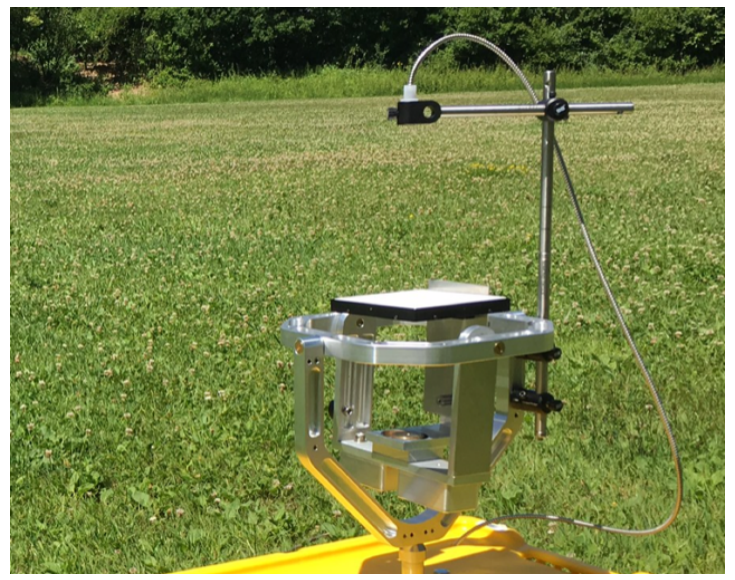

(b)

Fig. 1. Field spectroradiometer setup for irradiance measurements. Fig. 1a shows both instruments, one mounted on a combined sled/gimbal system and the reference system on a tripod and Fig. 1b shows a close-up onto the gimbal mount for the spectralon panel.

bare fibre optics (no for-optics were used) with a field of view of $25^{\circ}$ was positioned nadir looking, roughly $12 \mathrm{~cm}$ above the spectralon. This results in a footprint of $2.8 \mathrm{~cm}$ in radius. The second spectroradiometer was mounted onto a sled with the spectralon mounted on a passive gimbal with the fibre optics again positioned nadir looking, roughly $12 \mathrm{~cm}$ above the spectralon. With this setup, the spectralon itself was located $70 \mathrm{~cm}$ above the ground. The instrument setup can be seen in Fig. 1. Before and after the experiment, the two instruments were cross-calibrated by placing the sled mounted instrument next to the reference instrument and measuring the incoming irradiance with the two instruments at the same time for one minute.

The reference instrument on the sunlit patch ran throughout the whole experiment and acquired irradiance readings every five seconds. Several transects were acquired within the tree shadow with the spectroradiometer mounted on the sled, including the transition zone into the sunlit area. The transects were spaced $1 \mathrm{~m}$ apart from each other. Along the transects, five irradiance readings were acquired in short succession every $1 \mathrm{~m}$, once the gimbal was stable. In total, 13 transects in south-north direction were acquired to measure the irradiance inside and in the transition zone of the tree's cast shadow. Additionally to the shadow transects, seven radial transects along the major cardinal directions (NE, E, SE, S, SW, W, 


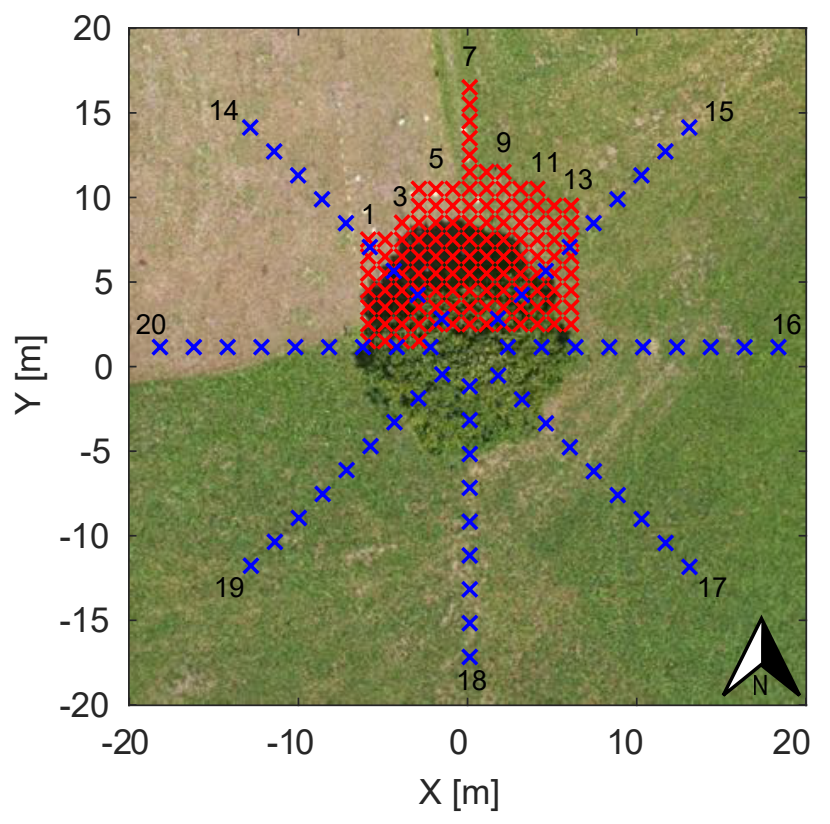

Fig. 2. Irradiance measurement pattern. Red: shadow transects (spacing: $1 \mathrm{~m}$ in $\mathrm{x}$ and y), blue: radial transects along major cardinal directions (spacing: $2 \mathrm{~m}$ ). Reference irradiance measurement location outside shown image at local coordinate $\mathrm{X}=-24, \mathrm{Y}=17$. Odd numbered shadow and all radial transects are labeled for referencing purposes. Background image acquired from drone data

NW) were acquired with a spacing of the individual irradiance readings of $2 \mathrm{~m}$. A detailed map of the measurement pattern with all measurement positions is shown in Fig. 2. The acquisition of all transects took 2 hours (11:16 until 13:06 UTC).

All field spectroradiometer data were stored in the spectral information system SPECCHIO to enable streamlined post-processing and ensure long-term data availability [39]. ASD spectroradiometers exhibit radiometric discontinuities at the joints between the three separate detectors used in the full-range instruments [40]. Environmental temperatures drive the magnitude of these radiometric steps and affect the whole spectral range to a higher or lower degree [41]. Such discontinuities were corrected at radiance level by applying a dedicated correction model to all spectra stored in SPECCHIO [41]. Ground reflectance factors were calculated in SPECCHIO using linear irradiance estimations [42].

In order to make the sunlit reference measurements and the transect measurements comparable, the transect measurements were first calibrated to the reference instrument by using the cross-calibration measurements from the beginning and the end of the experiment. A cross-calibration factor for each transect measurement was acquired by linearly interpolating between the two cross-calibration measurements. The five irradiance readings per target location were averaged to receive the final irradiance value for each measurement location. In order to decouple the irradiance changes caused by the tree from possible irradiance variabilities caused by subtle changes in atmosphere conditions or solar angle, target irradiance measurements were normalized using the reference irradiance measurements according to equation 3 .

$$
\bar{E}_{\text {target }}=\frac{E_{\text {target }}}{E_{\text {ref }}} \times 100
$$

where $\bar{E}_{\text {target }}$ is the normalized target irradiance, $E_{\text {target }}$ is the cross-calibrated target irradiance and $E_{r e f}$ is the reference irradiance measured at the sunlit reference location at the time of the acquisition of Etarget. 


\section{DART simulation validation}

For each measured transect one DART simulation was performed to ensure the same solar angles within the simulation and the measurements. To compare DART simulation outputs with the measurements, a 2D irradiance map was generated from the 3D total irradiance grid. As we measured the irradiance on a spectralon panel at $70 \mathrm{~cm}$ above ground, we extracted the irradiance value at this height above ground from the voxel grid. The starting position of each shadow transect relative to the northernmost point of the tree trunk has been measured in the field and can therefore be matched to a location on the DART irradiance output. The starting point of the radial transects was chosen to be $2 \mathrm{~m}$ away from the closest point of the tree trunk. All subsequent measurements were $1 \mathrm{~m}$ apart for the shadow transects or $2 \mathrm{~m}$ apart for the radial transects. In that way, we were able to locate the measurement location on the extracted irradiance map. However, as some geolocational errors can be expected with these relative locational measurements, the average irradiance as well as the standard deviation of a $3 \times 3$ pixel window $(75 \times 75$ $\mathrm{cm}$ ) around the identified measurement location was extracted from the DART irradiance map.

\section{Impact on Vegetation Indices}

The impact of irradiance effects on the retrieval of commonly employed vegetation indices was evaluated by comparing vegetation indices retrieved assuming a flat, homogeneously illuminated surface (apparent ToC reflectance) with indices retrieved from true ToC reflectance values. DART outputs apparent ToC reflectances by default. True ToC reflectance values were derived by dividing the apparent ToC radiance simulated by DART with the ToC irradiance values derived from the DART 3D radiative budget. This represents a perfect atmospheric correction where all illumination effects are accounted for, whereas the apparent ToC reflectance image represents the typical reflectance image uncorrected for illumination effects caused by surrounding objects. In order to decouple changes in the modeled reflectance and vegetation indices products due to varying irradiance from changes due to different ground materials, we parameterized the surface to be completely covered by grass with a single reflectance spectrum measured on the South-Eastern side of the tree.

Vegetation indices are an empirical approach to relate light measurements to vegetation information. However, wrong estimates of surface irradiance pose an additional sensitivity to these indices and complicate their interpretation, even if properly calibrated for a specific site, vegetation type and phenological period [6]. The choice in vegetation indices was based on those incorporating spectral information in the VIS/NIR wavelength regions since they are strongly affected by illumination effects and are of high importance in the functioning of vegetation. The chosen indices include the photochemical reflectance index (PRI) indicative for the de-epoxidation state of xanthophylls and often applied as a proxy for light use efficiency (LUE) [43]-[45] (Eq. 4). Additionally, we applied two indices sensitive to the relative content of chlorophyll and carotenoids as proposed by [46] (Eqs (5) and (6)):

$$
\begin{gathered}
P R I=\frac{r_{531}-r_{570}}{r_{531}+r_{570}} \\
C H L \propto\left[r_{540-560}^{-1}-r_{790}^{-1}\right] \times r_{790} \\
C A R \propto\left[r_{510-520}^{-1}-r_{560-570}^{-1}\right] \times r_{790}
\end{gathered}
$$

where $r_{x}$ denotes the reflectance at the specified wavelength $x$ in nanometers. The subscripts in Eqs. 5 and 6 indicate wavelength ranges used for the calculation of both indices. The wavelength subscripts $r_{x_{1}-x_{2}}$ denotes the average reflectance between $x_{1}$ and $x_{2} \mathrm{~nm}$. 


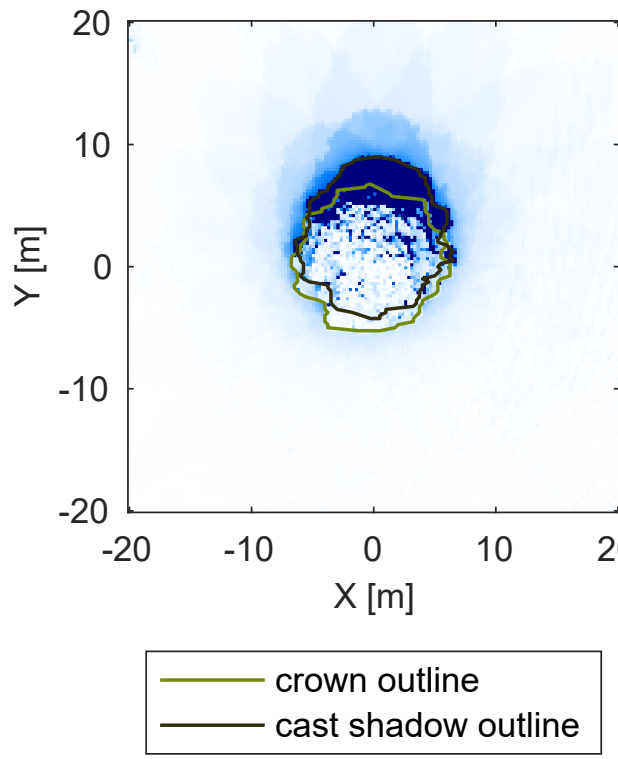

(a)
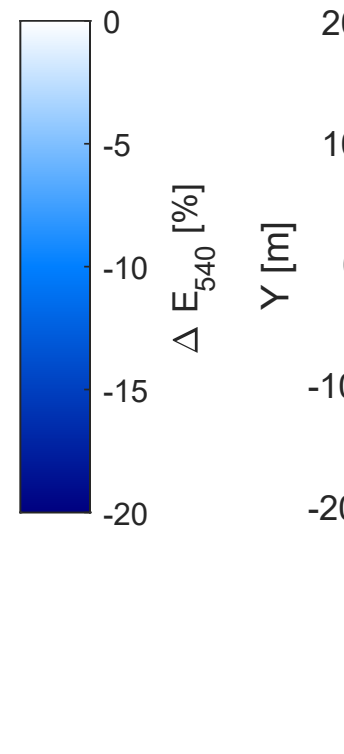

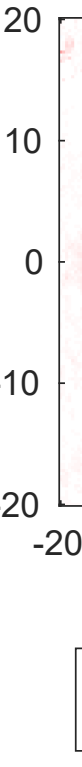

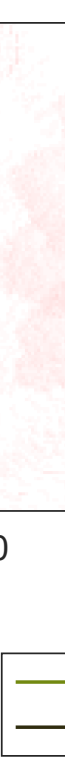

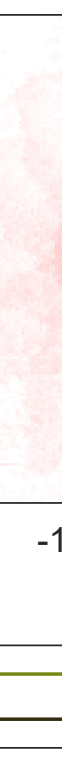

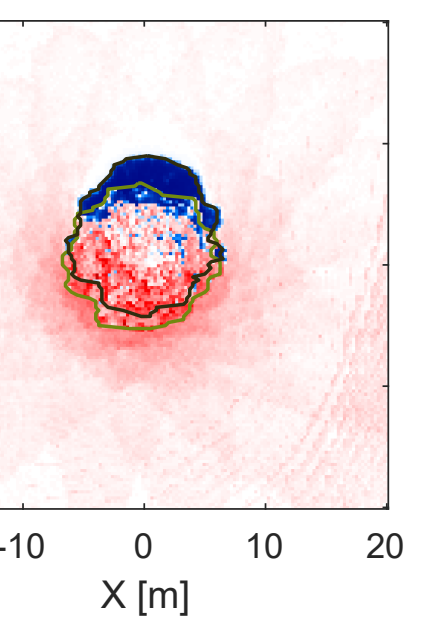

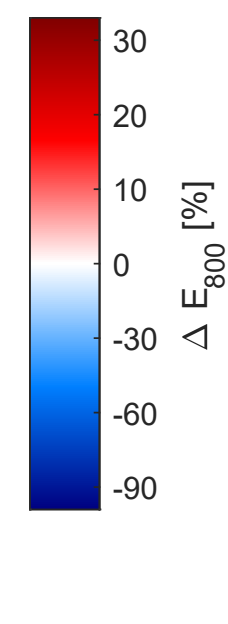
cast shadow outline

(b)

Fig. 3. ToC surface irradiance changes as modeled by DART at (3a) $540 \mathrm{~nm}$ and (3b) $800 \mathrm{~nm}$. Relative changes compared to modeled BoA irradiance are shown. Red colors denote an increase in irradiance due to the tree's presence, blue colors denote a decrease in irradiance due to the tree's presence. The tree crown outline and the cast shadow outline are marked with green and black lines respectively.

\section{RESUlts}

\section{A. Modeled Irradiance}

A total of 3560 DART simulations (20 solar positions corresponding to the transect acquisition times at 178 wavelengths with band width of $1 \mathrm{~nm}$ ) were evaluated to analyze the influence of the isolated tree on the surrounding irradiance pattern. An additional 1873 simulations were analyzed for the main SouthNorth shadow transect (transect \#7) for a spectrally higher resolved irradiance spectrum to be compared to the spectroradiometer measured irradiance. All simulations were performed on the cloud computing infrastructure ScienceCloud (S3IT) provided by the University of Zurich. Fig. 3 shows the ToC irradiance changes relative to the BoA irradiance for $540 \mathrm{~nm}$ and $800 \mathrm{~nm}$. Blue colors denote a decrease and red colors denote an increase in irradiance due to the presence of the tree. From Fig. 3 we can clearly distinguish an influence of the tree onto the surrounding irradiance field, also outside of the apparent cast shadow. At $540 \mathrm{~nm}$, the irradiance north of the tree a few meters $(1-2 \mathrm{~m})$ outside of the cast shadow is still 4-6\% decreased relative to the BoA irradiance. To the south of the tree, barely any influence of the tree on the surface irradiance is distinguishable at $540 \mathrm{~nm}$. On the other hand, at $800 \mathrm{~nm}$ the tree shows its biggest influence to the south of the tree, where the surface irradiance is increased by 6 to $7 \%$ even two meters away from the outer rim of the tree crown. The influence of the tree decreases with increasing distance, reaching less than $1 \%$ of irradiance increase at roughly $12 \mathrm{~m}$ away from the outer rim of the tree crown. The increase in irradiance is also visible on the southern side of the tree crown itself. The slight increase in irradiance at $800 \mathrm{~nm}$ in the South-Eastern corner of the image is not due to the tree but caused by the increasing slope of the terrain and the discrete voxel representation of the three-dimensional scene inside DART, where vertical voxel faces can illuminate or shadow lower neighboring voxels.

In Fig. 4, a vertical profile through the middle of the tree in the South-North direction is shown. This shows the brightening or darkening effect caused by the tree also in the third dimension and highlights the possibility to analyze vertical light availability and extinction profiles using DART. 


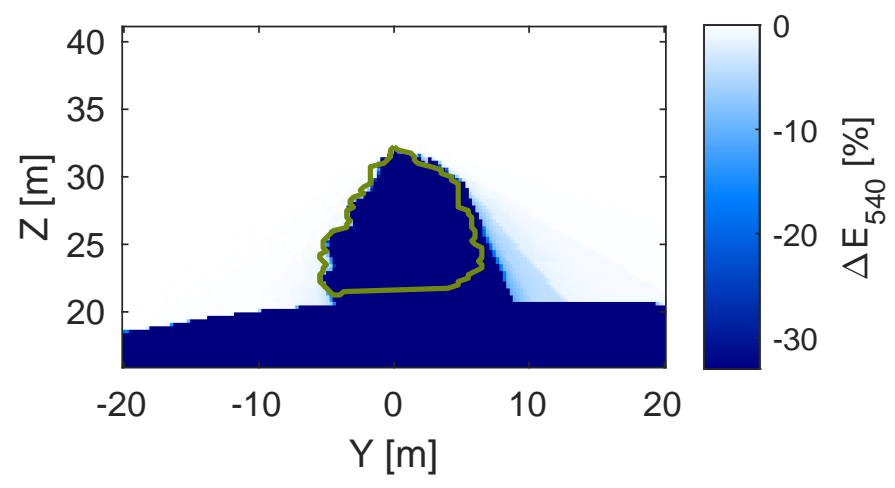

(a)

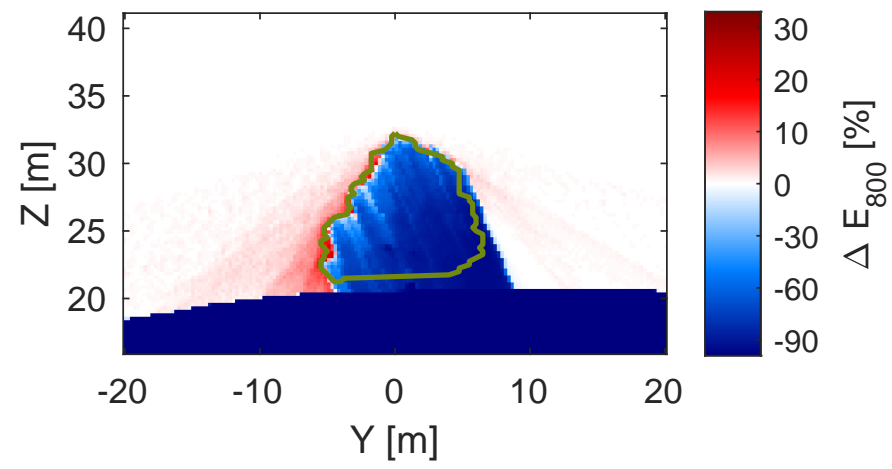

(b)

Fig. 4. Vertical DART profile of the modeled radiative budget in South - North direction. South is to the left of the figure. The crown outline is shown with a green line. Relative changes compared to the BoA irradiance are plotted as differences of $\Delta E$. Red colors denote an increase in irradiance due to the tree's presence, blue colors denote a decrease in irradiance due to the tree's presence. (4a) shows the vertical profile at $540 \mathrm{~nm},(4 \mathrm{~b})$ at $800 \mathrm{~nm}$.

\section{B. Measured Irradiance}

Fig. 5 shows the mean irradiance spectrum measured at the reference instrument over the whole measurement period as well as the irradiance time series for two wavelengths $(540 \mathrm{~nm}$ and $800 \mathrm{~nm})$. Except for a few local minima in irradiance most probably caused by high cirrus clouds blocking the direct solar path, the sunlit irradiance stayed relatively stable and shows a steady decrease after solar noon at 11:31 (UTC). Due to an instrument malfunction between 12:26 and 13:05 no reference irradiance measurements have been acquired and the data gap was filled using linear interpolation. A total of five transects are affected by this measurement gap (radial transects 15 to 19). However, the error introduced due to the missing reference irradiance is assumed to be small (i.e. $+/-3.26 \%$ in the VIS, $+/-3.5 \%$ in the NIR, and $+/-6 \%$ in the SWIR region, based on the standard deviation of the measured reference irradiance 15 minutes before and after the measurement gap). Furthermore, a large decrease or increase in solar irradiance would be visible in the transect measurements allowing for an identification of the discrepancy between interpolated reference irradiance and actual irradiance. The main analysis performed in this study is focused on the shadow transects (transect numbers 1 to 13) for which the reference irradiance time series is complete. The only exceptions are transects 18 (North - South transect) and 19 (North-East - South-West transect) showing the irradiance fields on the southern side of the tree. A linear interpolation of the data gap is a simplifying solution for this problem. A better model to describe the irradiance change over the course of a day would include Lambert's cosine law and reflect the change in airmasses also leading to a non-linear decrease in transmittance of the atmosphere. However, given the date, time of day, length of the experiment as well as the latitude, the error introduced by using a simple linear interpolation is assumed to be minor. 


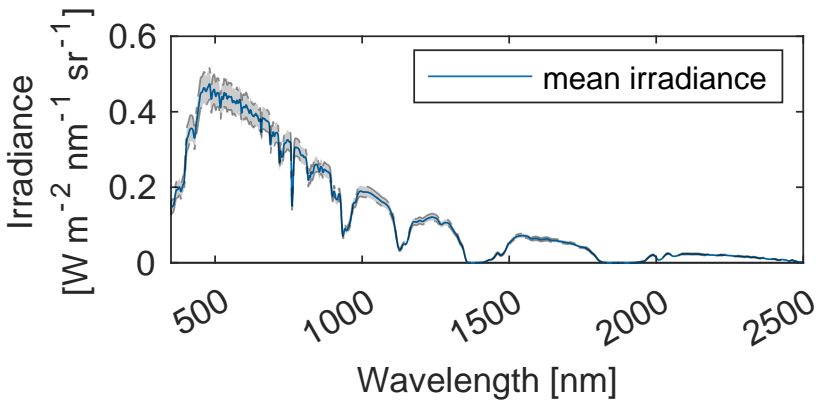

(a)

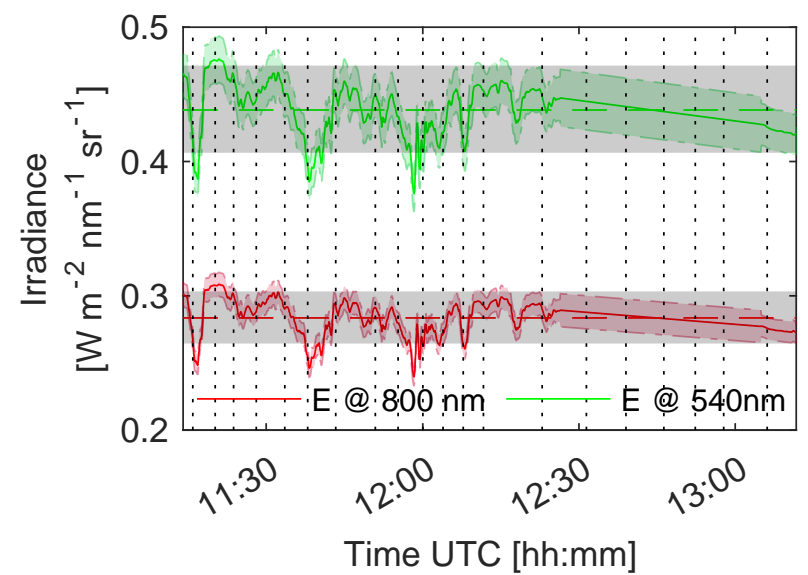

(b)

Fig. 5. Sunlit reference irradiance measurements. (5a) shows the mean irradiance spectrum measured with the reference instrument. The gray area denotes the standard deviation over the whole measurement period plus the uncertainty associated with the irradiance measurements. (5b) shows the reference irradiance time series over the whole measurement period at 540nm (green) and $800 \mathrm{~nm}$ (red). The green and red transparent area denotes the uncertainty associated with the irradiance measurement. The mean irradiance for the respective wavelength is shown with a dashed line. The gray boxes denote the measurement uncertainty and the $+/$ - standard deviation over the whole measurement period for the respective wavelength. The gray dotted vertical lines denote the start time of a new transect. For transects 15 to 19 , reference irradiance values have been interpolated due to an instrument malfunction. The uncertainty introduced due to the interpolation of the data gap was estimated based on the irradiance variability 15 minutes before and after the gap and added to the total uncertainty associated with the irradiance measurements for the interpolated irradiance values.

Fig. 6 shows the irradiance of transect numbers 7 and 18 (South-North transect in the middle of the tree and North-South transect on the Southern side of the tree) at $540 \mathrm{~nm}$ and $800 \mathrm{~nm}$. Fig. 6 clearly shows the wavelength dependent behavior of the irradiance with increasing distance from the tree. The first few measurements were located inside the cast shadow of the tree, followed by a small transition zone and a longer sunlit area. The irradiance at $540 \mathrm{~nm}$ increases inside the cast shadow caused by the gain in sky view factor with increasing distance from the tree. The visible range of the spectrum and especially the blue spectral range is highly influenced by atmospheric scattering. A reduced sky view factor therefore further decreases the diffuse atmospheric irradiance component on the ground, which is still observable outside of the apparent cast shadow. As wavelengths in the near-infrared region are less affected by atmospheric scattering, this effect is not visible in the cast shadow at $800 \mathrm{~nm}$. On the southern side of the tree a different effect is visible, where the irradiance at the border to the cast shadow spikes at $800 \mathrm{~nm}$ and then decreases again with increasing distance from the tree. This can be explained by an additional diffuse irradiance component caused by the high backwards scattering characteristics of vegetation in the near-infrared region. This irradiance increase in close vicinity of the tree can be observed in all transects facing towards the sun. This effect is not apparent in the visible domain as shown in Fig. $6 c$. 


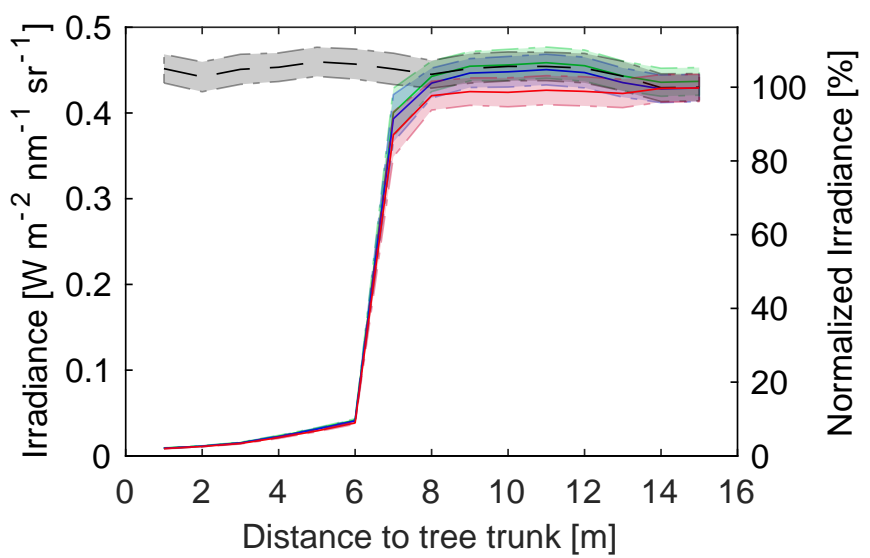

(a)

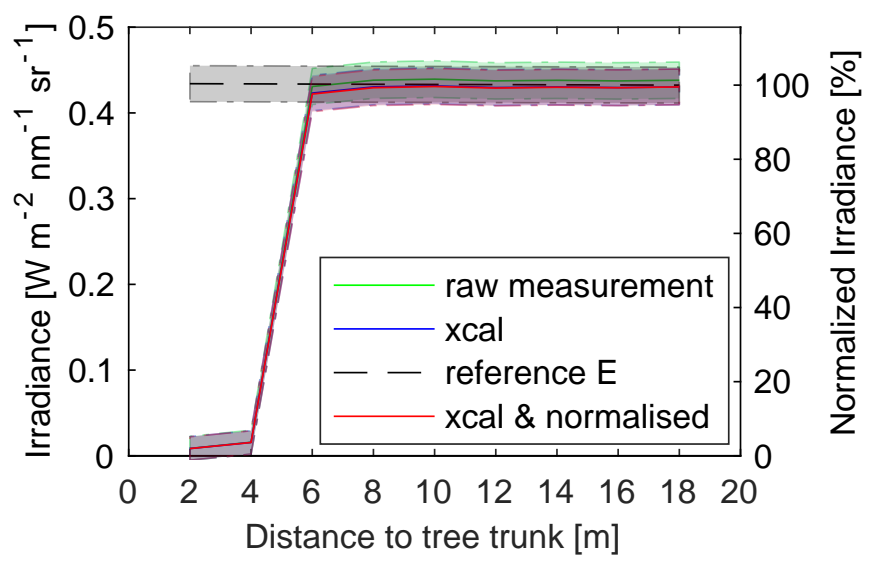

(c)

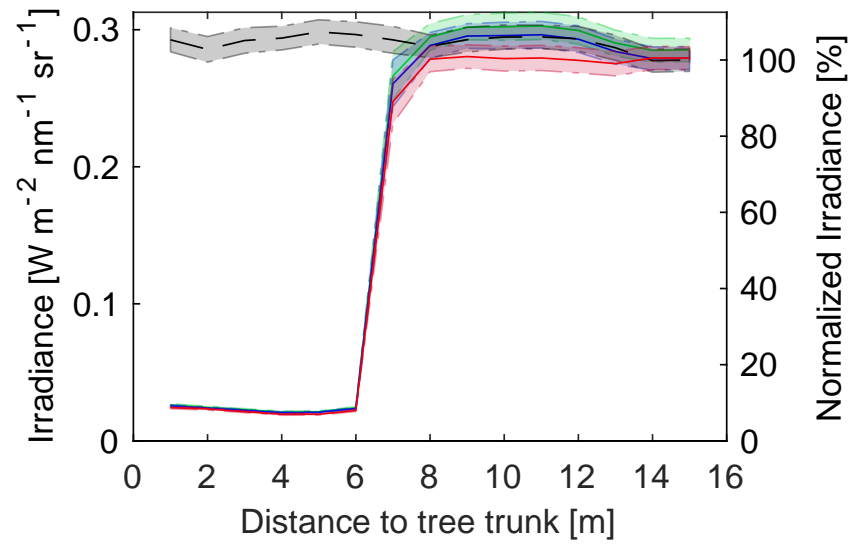

(b)

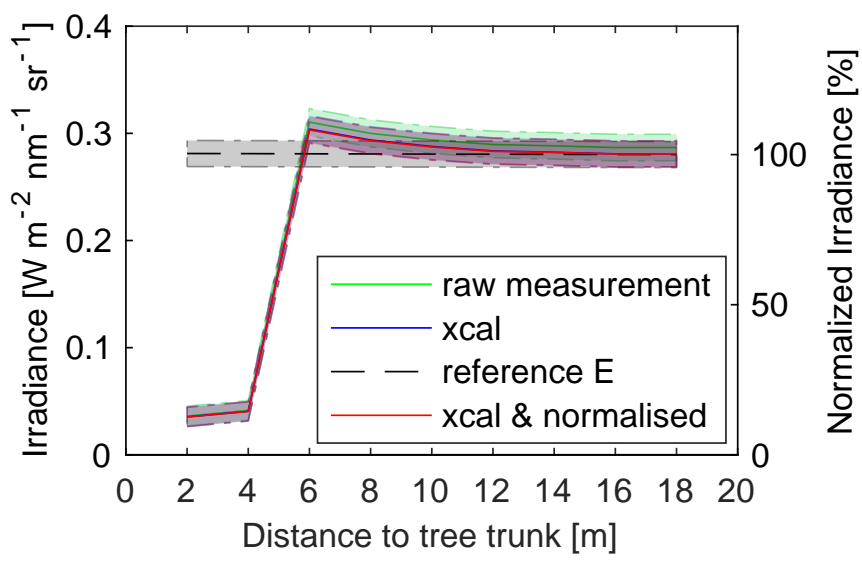

(d)

Fig. 6. Measured irradiance for transect \#7 and \#18 at 540nm (6a and $6 \mathrm{c}$ ) and at $800 \mathrm{~nm}$ ( $6 \mathrm{~b}$ and $6 \mathrm{~d}$ ). Measured raw irradiance in green, measurements cross-calibrated with reference instrument in blue and cross-calibrated plus normalized measurements in red. Normalization has been performed according to the irradiance at the reference instrument during the target acquisition. The irradiance at the reference instrument is shown using a dashed black line (reference E). Shaded area in respective colors denote the uncertainty associated with the irradiance measurement plus the standard deviation of the five irradiance readings per target. Note that the reference irradiance values for transect 18 (Figures 6c and 6d) have been interpolated.

\section{DART simulation validation}

Figures 7 and 8 show the comparison between simulated and measured irradiance averaged over all sunlit and shadowed targets for transect \#7 both in absolute and normalized numbers. Normalization in Fig. 8 is based on the measured reference irradiance at the time. DART generally overestimates the surface irradiance compared to the measured irradiance. In the visible spectral range $(350-700 \mathrm{~nm})$ the overestimation is at $12 \%$ on average and $9.3 \%$ in the near infrared $(701-1000 \mathrm{~nm})$ for sunlit targets. In the short-wave infrared $(1001-2500 \mathrm{~nm})$ the average overestimation is at $10.9 \%$ (wavelength inside absorption features highlighted in gray and green in Fig. 7 and Fig. 8 were excluded from these statistics). For the shadowed targets DART overestimates the surface irradiance by $37.4 \%$ in the visible range. For the NIR and SWIR region, DART underestimates the surface irradiance in average by 1.5 and $16.5 \%$. However, due to the large variability in irradiance in the shadowed areas, the simulated and measured irradiance values lie within their respective uncertainty bounds (standard deviation of all shadowed and sunlit targets of transect \#7 for modeled irradiance, uncertainty plus standard deviation of all shadowed and sunlit targets of transect \#7 for measured irradiance).

The overall good fit between DART simulated and spectroradiometer measured irradiance patterns with 


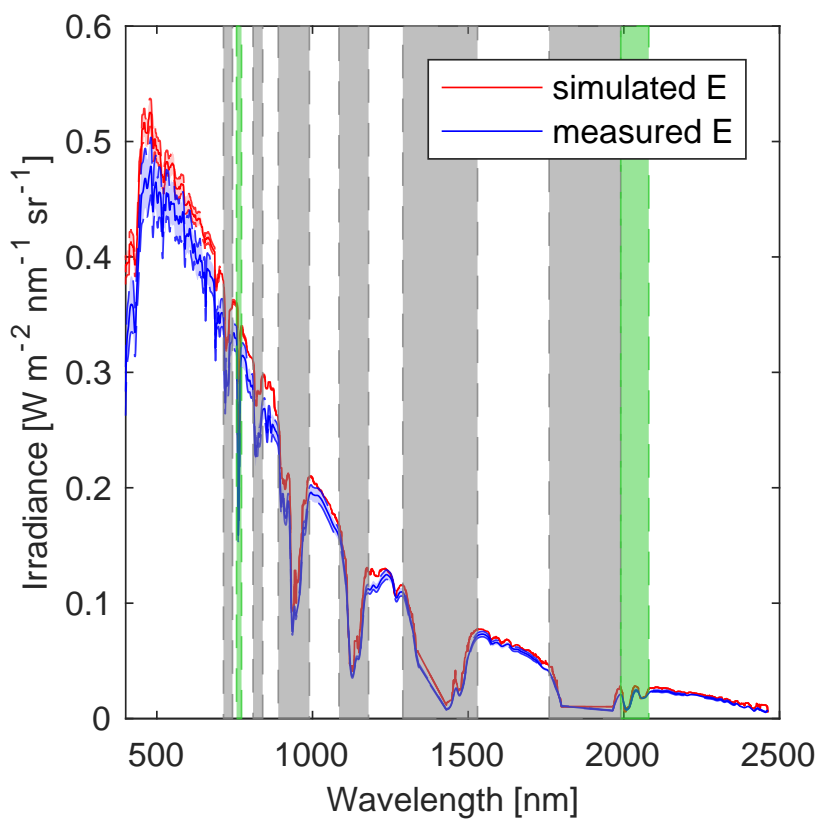

(a)

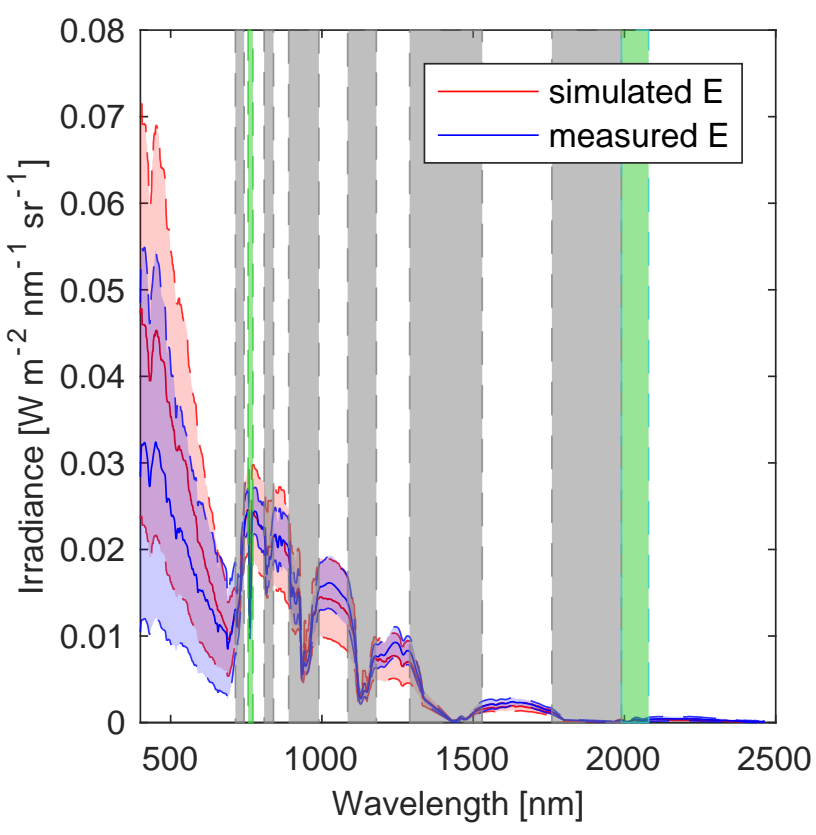

(b)

Fig. 7. Average irradiance spectra for sunlit (7a) and shadowed (7b) targets of transect \#7. Simulated irradiance in red and measured irradiance in blue. Standard deviation over all sunlit and shadowed modeled irradiance in transparent red areas. Standard deviation over all sunlit and shadowed measured irradiance plus uncertainty associated with the spectrometer irradiance measurements in transparent blue areas. Absorption bands due to water vapor or atmospheric gases are highlighted in gray or green columns respectively.

an $R^{2}$ of 0.997 can also be seen in Fig. 9, where the absolute measured and modeled surface irradiance are plotted against each other. The 1:1 line shown in Fig. 9 also highlights the slight overestimation of DART modeled irradiance.

When analyzing single transects at selected wavelengths, we observe a strong agreement in ToC irradiance patterns between measured and modeled ToC irradiance. Fig. 10 shows that the stated darkening effect of the tree on the northern side in the blue spectral range as well as the brightening effect of the tree especially on the southern side in the near infrared region are both observable in the measured as well as the modeled surface irradiance pattern.

\section{Impact on Vegetation Indices}

The results shown above illustrate the impact of vegetation on the surrounding irradiance field. Related uncertainties will translate to the calculation of higher level remote sensing products such as vegetation indices or vegetation biochemical properties and compromise their reliability. Selected vegetation indices (i.e. PRI, CHL, CAR) were derived using true and apparent ToC reflectance values to quantify the impact of illumination effects on retrieved vegetation information. In Fig. 11 the difference between PRI, CHL, and CAR derived from apparent and true ToC reflectance values is shown relative to the value range observed for the respective vegetation products derived from the measured leaf and ground optical properties (see Section III-A3). Red colors therefore denote an increase of the respective vegetation index value when the presence of the tree is not considered in the calculation of ToC reflectance. On the other hand, blue colors denote a decrease of the respective vegetation index values when the processing neglects the presence of the tree. These changes can be observed outside (i.e. up to $10 \mathrm{~m}$ distance) the apparent cast shadow. CHL and CAR show an overestimation of up to 10-14\% (relative to the observed value range of the respective vegetation product derived from the leaf and ground optical properties measurements) close to the edge of the tree and the cast shadow if only apparent ToC reflectance values are used to derive these vegetation products. At $4 \mathrm{~m}$ distance from outer rim of the tree crown and cast shadow, the 


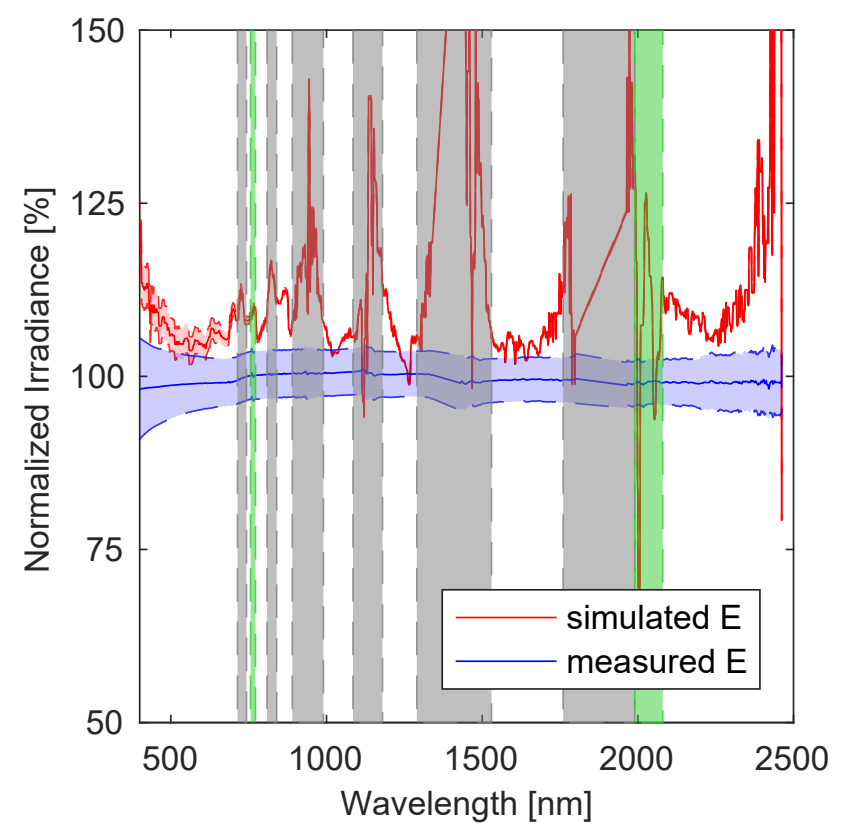

(a)

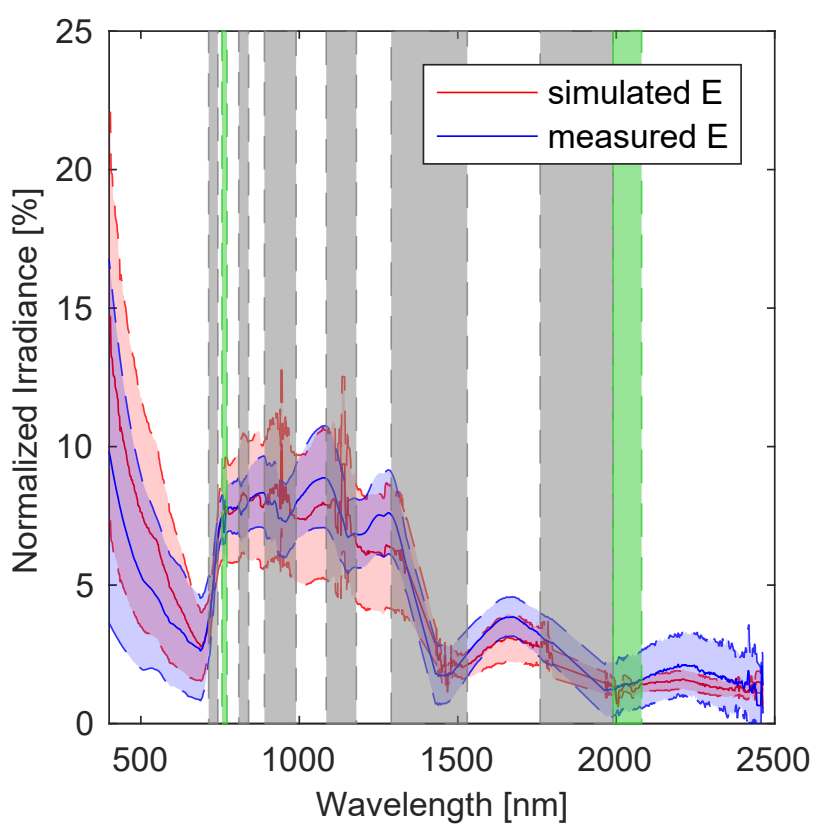

(b)

Fig. 8. Average normalized irradiance spectra for sunlit (8a) and shadowed (8b) targets in transect \#7. Simulated irradiance in red and measured irradiance in blue. Standard deviation over all sunlit and shadowed modeled irradiance in transparent red areas. Standard deviation over all sunlit and shadowed measured irradiance plus uncertainty associated with the spectroradiometer irradiance measurements in transparent blue areas. Normalization based on the irradiance values measured at the reference instrument location. Absorption bands due to water vapor or atmospheric gases are highlighted in gray or green columns respectively.

overestimation is still up to $6 \%$. As both CHL and CAR rely on reflectance values in the NIR spectral domain, they also show a slightly larger impact on the southern side of the tree due to the pronounced backscattering characteristic of the tree in the NIR region. By relying on reflectance values in the green spectral range, PRI shows a contrary behavior, where a larger influence on the northern side of the tree is discernible. Due to the employed wavelength for the calculation of the PRI, the general difference between the PRI derived from true and apparent ToC reflectance is contrary to the other vegetation indices. PRI inside the cast shadow is overestimated by up to $90 \%$ of the observed PRI range derived from the leaf and ground optical properties measurements when using apparent ToC reflectance to calculate PRI. PRI outside the cast shadow is slightly underestimated by up to $5 \%$ when using apparent ToC reflectance. The PRI outside the cast shadow is therefore much less affected by the presence of the tree than the other discussed vegetation indices. However, due to the very narrow wavelength region used to calculate the index $(531 \mathrm{~nm}$ and $570 \mathrm{~nm})$ and the thus inherent narrow value range of the PRI close to 0, the stated relative values have to be taken with caution.

\section{Discussion}

\section{A. Modeled Irradiance}

Due to the numerous parameters used by DART to simulate the experimental site and the atmosphere, several sources of uncertainty for the modeled irradiance values can be identified, e.g. vegetation density per voxel (i.e. plant area density PAD), provided leaf optical properties (LOP), or the leaf angle distribution. The AMAPvox software used to estimate PAD per voxel from TLS acquisitions tends to overestimate Plant Area Index (PAI) values obtained from vertical integration of PAD profiles as compared to PAI estimates derived from average gap fractions using LAI2200 measurements in a tropical forest [34]. Observed differences, however, reflect the fact that distribution of foliage is strongly spatially structured which is not properly accounted for in PAI estimates derived from mean gap fraction per elevation angle 


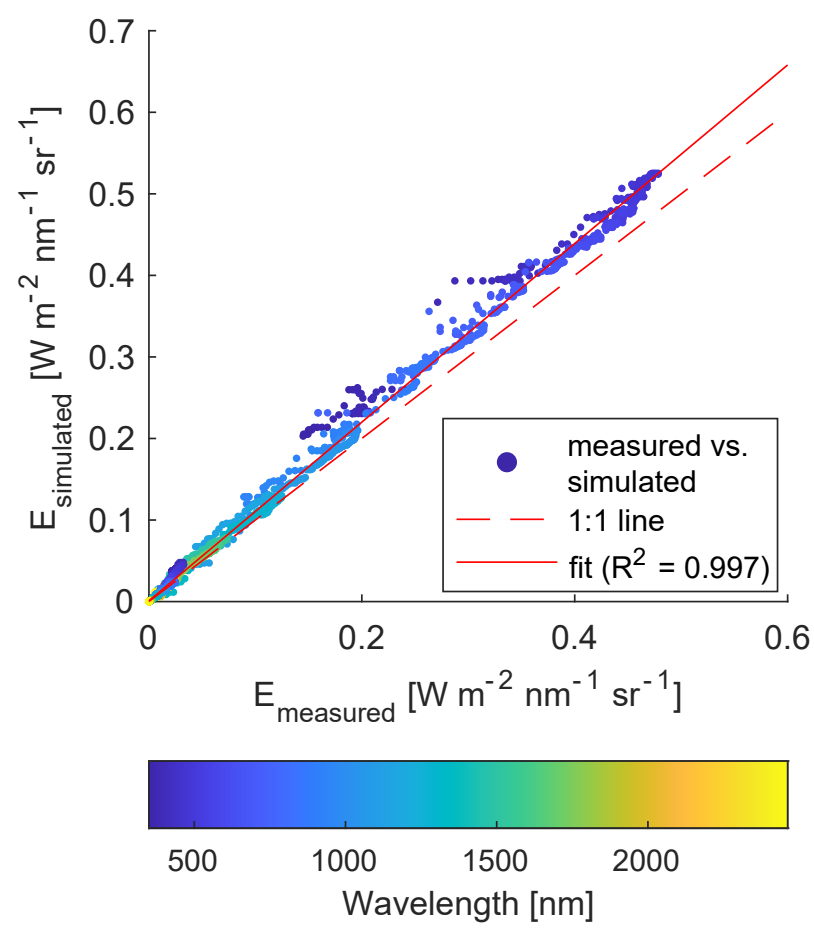

Fig. 9. Measured vs. simulated irradiance scatterplot for transect \#7.

as performed with the LAI2200 instrument [34]. However, TLS derived PAD and PAI estimates using similar approaches as the one in AMAPvox showed good results (e.g. estimated leaf area lay within 14\% from the reference measurement acquired by leaf harvesting for a savanna environment as reported in [47]) rendering this approach superior to previously established methods using optical measurements often not able to vertically resolve the PAD distribution in the canopy e.g. [47]-[50].

Leaf optical properties are recognised as one of the major components driving the radiation regime of forest canopies. Thus LOP are also key input parameters for leaf and canopy radiative transfer models [51], [52]. It is therefore of great importance to know the uncertainties associated with the LOP measurements. Especially LOP retrieved from leaf clip measurements are known to have differing reflectance and transmittance when compared to the well established procedure using a single integrating sphere [53]. However, the assessment of the uncertainty associated with the LOP measurements is a nontrivial task. Nevertheless, we assume that even larger errors associated with the LOP measurements will not significantly impact the modeled surface irradiance values and patterns and therefore the findings of this study. This assumption is also based on the findings by Stuckens et al. [20], where they analysed commonly used assumptions in radiative transfer models such as the averaging of LOP for entire crowns. They found that no measurable error could be found in the simulated reflectance images if an averaged LOP was used instead of a more accurate assignment with varying LOP throughout the crown. A more detailed analysis on the uncertainty associated with the measured LOP and the impact on simulated surface irradiance is given in Appendix A-E.

Also leaf angle distribution (LAD) plays an important role in the radiative transfer through tree canopies [54], but also in the estimation of PAD per voxel [47]. As an automatic estimation of LAD from TLS data is still a challenge, we assumed a spherical leaf angle distribution for the whole crown. This assumption is commonly performed in literature e.g. in [34], [55], [56] and was reported to be acceptable for mapping effective LAI in a heterogeneous mixed forest from aerial discrete return lidar in [57]. We further had leaf angle measurements from a different tree of the same species available (Pisek, Tartu Observatory, unpublished data), proving the assumed spherical leaf angle distribution to be adequate. 

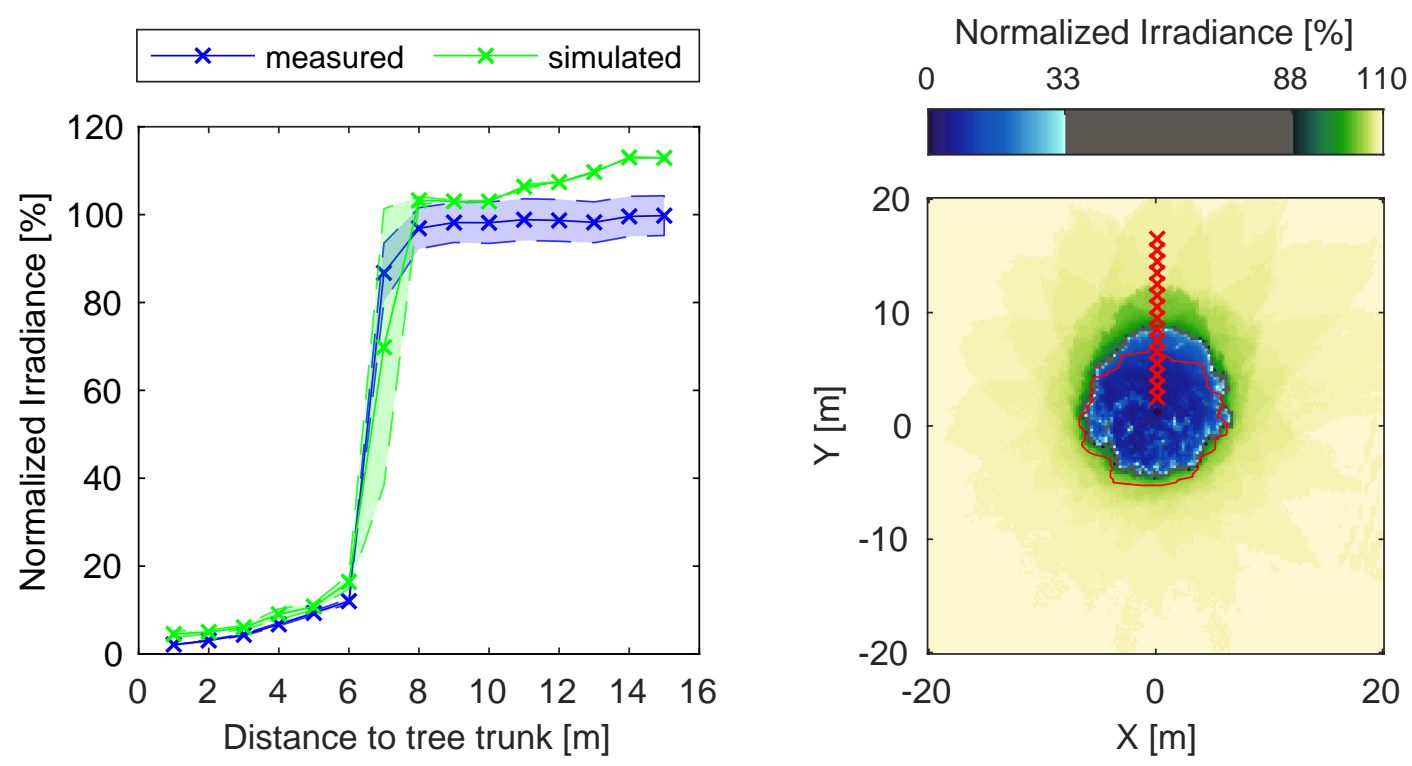

(a)
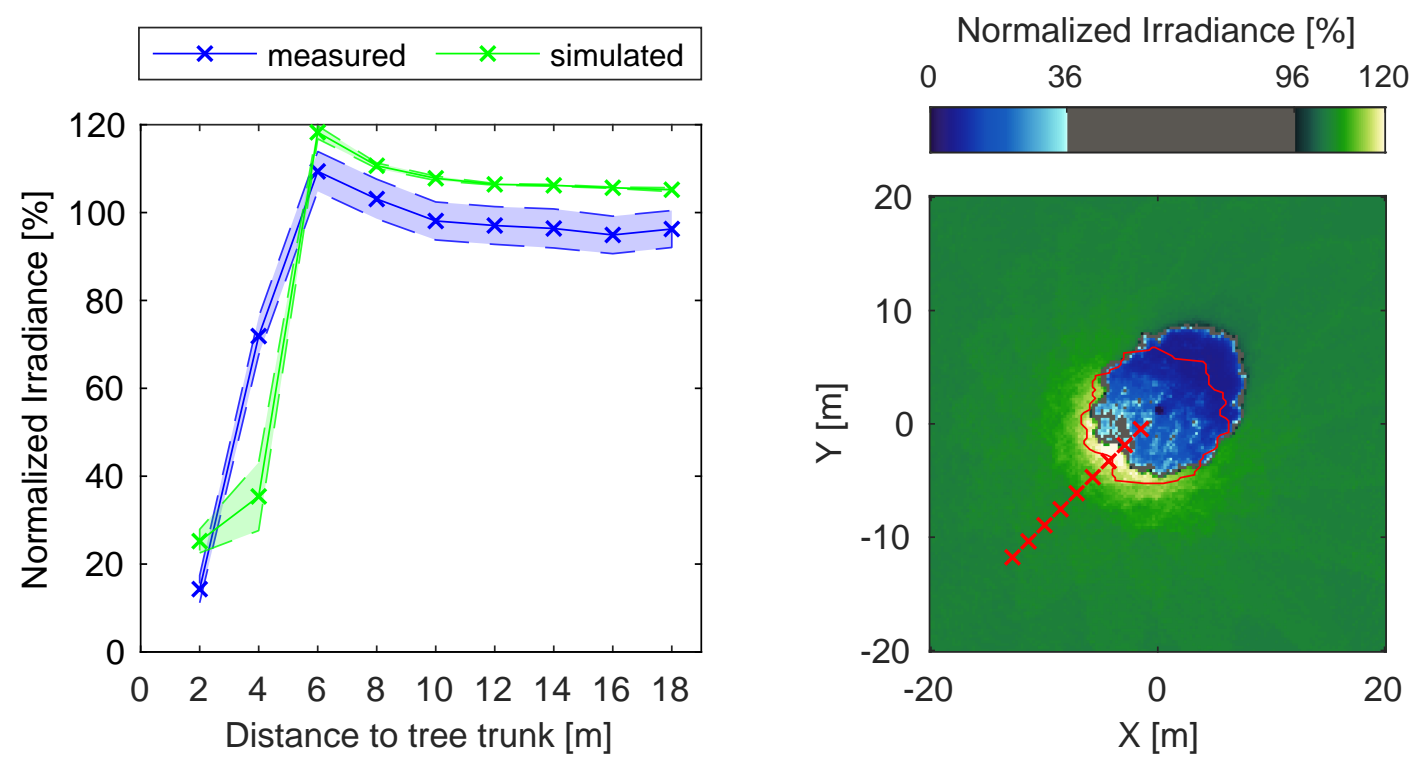

(b)

Fig. 10. Measured vs. simulated irradiance for transects \#7 (10a) at 470nm and \#19 (10b) at 781nm. Left panels show the normalized irradiance transects for measured (blue) and simulated (green) irradiance. Irradiance normalization has been performed based on measured irradiance at the reference location. Right panels show DART simulated normalized irradiance at $70 \mathrm{~cm}$ above ground with the crown outline and target locations highlighted in red.

No measurements of the atmospheric composition during the experiment are available, hindering an accurate, time-dependent parameterization of the atmosphere. We used the DART standard aerosol model for rural areas as a stable reference. The visibility was set to $23 \mathrm{~km}$, which is the value usually observed for clear summer days in Switzerland. The gas model which describes the vertical distribution of gases in the atmosphere and its scattering characteristics was defined as the DART standard mid-latitude summer model. In order to reduce this source of uncertainty, additional measurements using a sun-photometer should be used in future experiments. The temporal variability in solar irradiance further require temporally high resolved measurements of the atmospheric composition in order to fully reflect the influence of the atmosphere, adding even more to the complexity. We therefore opted for the use of a simple DART 


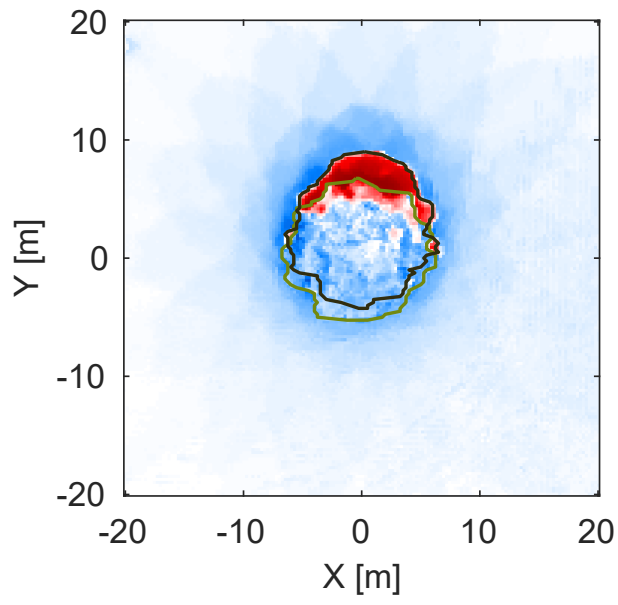

(a)

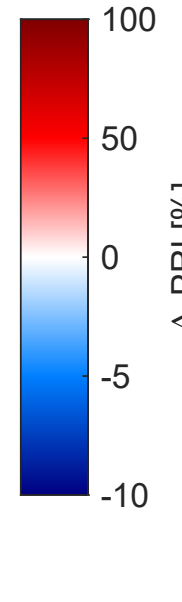

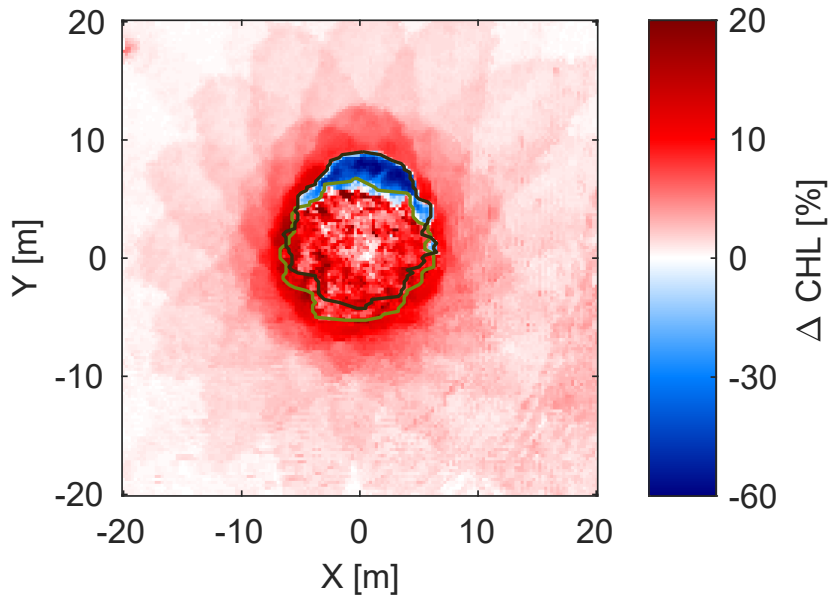

(b)

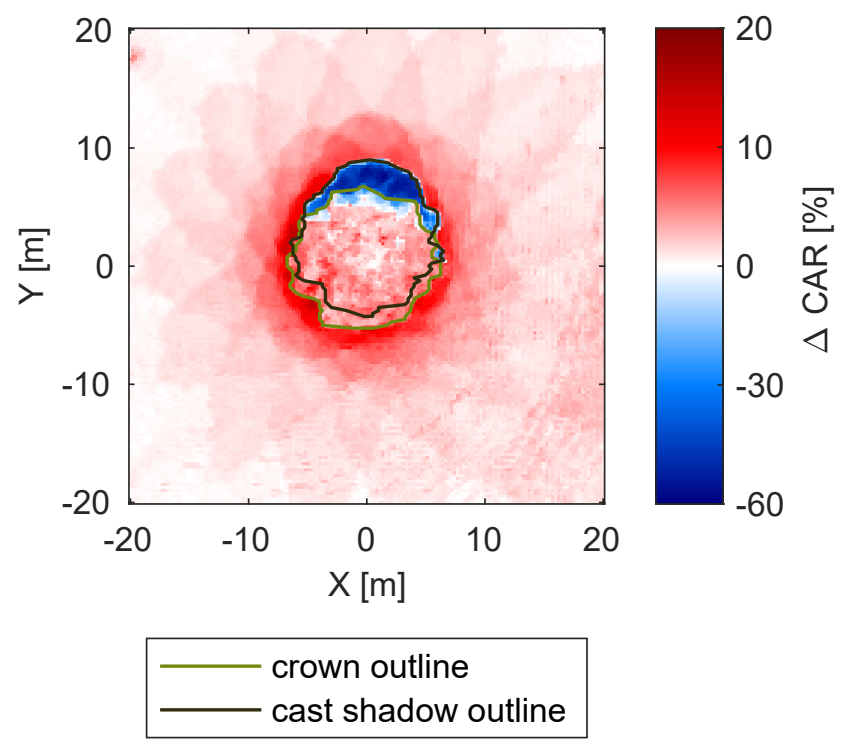

(c)

Fig. 11. Impact of the tree onto the retrieval of commonly employed vegetation indices. Red (blue) colors denote an overestimation (underestimation) of the derived product when not accounting for the tree's presence (i.e. when using apparent ToC reflectance values). $\triangle C A R=a C A R-t C A R$ where $a C A R$ is the vegetation index derived using apparent ToC reflectance values and $t C A R$ is the vegetation index derived using true ToC reflectance values. The changes are shown relative to the observed value range of the respective vegetation indices derived from the leaf and ground optical properties (c.f. Section III-A3).

standard atmosphere parameterization, which is applicable for most nearly clear sky situations and is a common approach found in literature e.g. [6], [58]-[60]. We consider this source of uncertainty not to impact the main findings of our study and drawn conclusions.

Several radial lobes around the tree can be distinguished in the DART derived irradiance maps. This is caused by the user defined discrete scattering directions in which DART is able to scatter light [61]. In all performed simulations a total amount of 100 directions in the whole $4 \pi$ space was defined plus an additional 100 directions in the sun's hot-spot up- and downward direction. In order to remove these lobes, the amount of directions has to be increased at the cost of computational expense. Again, this undersampling does not impact our results and conclusions. 


\section{B. Measured Irradiance}

Even though we used the field spectroradiometer measurements as a source for validating DART modeled irradiance, multiple sources of uncertainties associated with the irradiance measurements from the spectroradiometers have to be accounted for (cf. [42] for a comprehensive review of sources of uncertainties). An in-depth analysis on the uncertainty budget for the irradiance measurements is given in Appendix A. The biggest source of uncertainty associated with the irradiance measurements is believed to be found in the spectralon panel, especially due to degradation effects of the spectralon panel over time (see Appendix A-B), but also due to dirt build up during the experiment. The spectralon panels suffer from a gradual degradation over time which is most pronounced in the blue and visible spectral range. Möller et al. [62] showed that over the course of only one year, a degradation of up to $1 \%$ in reflectance can be observed depending on the wavelength. They even showed that over the course of a single day a degradation of the reference panel can be observed. Hueni et al. [42] also showed and quantified the panel degradation by comparing the measured reflectance to a new, pristine panel. They showed that the degradation affected the whole spectral range with the visible range being the most susceptible, showing degradation of up to $20 \%$ below $400 \mathrm{~nm}$. Above $600 \mathrm{~nm}$ the degradation was reported to be less than $5 \%$. We argue that this is one of the main reasons for the overestimation of the DART modeled irradiance values. However, the influence of the aging effect can be reduced with proper storage and could be quantified by frequently calibrating the spectralon panels with a pristine and calibrated reference spectralon panel that is ideally only used in laboratory environment. Unfortunately, such a calibration was not performed for this study due to a missing pristine and calibrated spectralon panel.

\section{DART simulation validation}

Even though a fixed measuring grid with the tree trunk and the major cardinal directions as a reference was laid out, the exact localization of each irradiance measurement location in the DART simulated irradiance products is likely a source of uncertainty in this experiment. To overcome this issue, the average simulated irradiance in a $75 \times 75 \mathrm{~cm}$ area around the localized measurement point was taken and the standard deviation inside this window was calculated. Nevertheless, an inaccurate localization of the measurement location inside the DART irradiance product could still be an explanation for some larger discrepancies between modeled and measured irradiance found especially at the shadow borders.

The results have shown that even though the irradiance pattern between measured and modeled irradiance fit well, the DART simulated values usually overestimated the measured irradiance. A reason for this overestimation, as already mentioned in Section V-A, could be the parameterization of the atmosphere inside DART. As seen in Fig. 5b, also minor changes in atmospheric conditions in a seemingly bright and sunny day can cause a significant variation of irradiation during a short time window (i.e. up to $10 \%$ depending on wavelength) on the measured baseline irradiance. Also differences in observed and modeled extra-terrestrial solar irradiance could have an influence on the modeling performance of the surface irradiance. However, the impact of such differences in extra-terrestrial solar irradiance is small and not accountable to the overestimation in modeled irradiance.

Because DART has many input parameters, each one with its own uncertainty, a whole uncertainty budget for the DART modeled irradiance is believed to be out of the scope of this study. Nevertheless, a quantified uncertainty budget for the DART modeled irradiance including several sensitivity studies for the input parameters is still needed and should be considered for future studies.

However, measured irradiance could have an influence on the observed overestimation too. As mentioned in Section V-B, spectralon panels degrade over time, especially at lower wavelengths. This could be a further explanation for the larger overestimation in modeled irradiance, especially below $500 \mathrm{~nm}$.

\section{Impact on Vegetation Indices}

The presence of a tree causes a wavelength dependent impact on surface irradiance of up to $7 \%$ that directly translates to retrieved $\mathrm{ToC}$ reflectance and subsequently calculated vegetation indices. It was found 
that this effect can be even more pronounced for vegetation indices using the near-infrared and the visible domain, such as the chlorophyll index or the carotenoid index [42]. Fig. 11 shows that an overestimation of up to $14 \%$ in CAR or CHL can be expected when we use apparent ToC reflectances to calculate the indices instead of actual target reflectance values. Even though this may not sound as much, it is of high relevance. Fox et al. [63] stated in their paper on the Traceable radiometry underpinning terrestrial- and helio-studies (TRUTHS) instrument that for long-term climate studies we need less than $1 \%$ uncertainty. Therefore, a tree induced irradiance change of $7 \%$ for a single wavelength or a change of $14 \%$ for a vegetation index is relevant. Already Roberts et al. and Roberts [18], [19] reported a similar halo effect especially pronounced in the near-infrared, where the downward radiant flux measured at a certain distance from the leaf was increased by up to $20 \%$.

Figures 3 and 11 show a brightness gradient for ToC irradiance and vegetation indices across the tree crown. These effects are especially pronounced for the irradiance field in the NIR spectrum as well as in the vegetation indices containing wavelengths in the NIR spectrum (CAR and CHL). This has serious implications for the retrieval of biochemical and biophysical parameters as well as of vegetation functioning from imaging spectroscopy data. For fully illuminated parts of the crown, oriented to the sun (i.e. an illumination angle close to 0 degrees) vegetation indices show errors of up to $2.5 \%, 10.6 \%$, and 4.8\% for PRI, CHL, and CAR respectively. For shaded crown pixels oriented away from the sun and mainly receiving diffuse irradiance, errors are up to $30 \%, 10.6 \%$, and $8.9 \%$ for PRI, CHL, and CAR respectively. These findings suggest that the robustness of commonly employed approaches that only take sunlit pixels to derive these parameters (e.g. [4], [64]) is compromised. Our findings suggest that only the flat area on the very top of the tree (i.e. the area that represents the assumed illumination conditions in terms of illumination angle and fraction of diffuse to direct irradiance) can be assumed to be unaffected by these adjacency effects. How these effects scale with increasing pixel size and related varying contributions of isotropic, volumetric and geometric-optical scattering has to be determined. Unfortunately, measuring the actual irradiance on the crown surface is a complicated task, making the validation of the modeled irradiance values on the crown surface and the retrieval of vegetation indices of such pixels a challenge. Nevertheless, such adjacency effects are likely often neglected and a more in-depth analysis of these effects also at the crown level and at different scales is needed in order to fully understand their influence on the retrieval of vegetation status and functioning. In addition, induced adjacency effects have a direct implication for the assessment of sub-pixel material abundances: Multiple scattering caused by illumination effects determine the mixing of spectral signatures non-linear and thus introduce uncertainties in estimated unmixing results (cf. [65]-[69]). Radiative transfer models such as DART are valuable tools to analyze and quantify these very complex adjacency effects due to anisotropy, absorption and multiple scattering in the vegetation canopy. The implemented modeling approach reveals that DART is able to accurately model such irradiance patterns and could therefore be used to analyze these complex light interactions in even more detail.

In this study, only the irradiance change induced by a single isolated tree was analyzed and discussed. However, Fawcett et al. [7] observed substantial border effects around tree crowns and concluded that the irradiance field is even more complex in presence of several trees due to multiple scattering effects. Also Stuckens et al. [20] confirmed a significant change in simulated scene reflectance for varying placements of citrus trees in an orchard. For future studies it is of interest to analyze the impact on the irradiance field caused by multiple trees and the light interactions between the trees, possibly giving insights on species co-existence strategies. The DART radiative transfer model could be a useful tool to analyze the radiative transfer through the forest canopy, describing the light interaction inside the canopy. This has further implications for large scale ecosystem demography models for which Fisher et al. [70] identified the radiative transfer through the canopy as one of the biggest sources of uncertainty.

\section{CONCLUSION AND OUTLOOK}

We conclude on the significance of spatial and spectral irradiance variability caused by $3 \mathrm{D}$ objects and the need to accurately model irradiance fields for reliable retrievals of geophysical information from 
imaging spectroscopy data. Measured and simulated irradiance demonstrate that shadow is a non-linear phenomenon showing a large variability in irradiance of up to $560 \%$ for blue wavelengths (450nm) and up to $17 \%$ for red wavelengths $(680 \mathrm{~nm})$. However, beyond the visible spectral range, the variability of irradiance inside the cast shadow is negligible. Isolated trees substantially influence the irradiance field of its surrounding that goes far beyond the apparent cast shadow. In certain wavelength regions, even the surrounding at the sun-facing side is substantially impacted.

Our findings showed that the presented modeling approach is able to simulate and predict the extremely complex interactions of radiation with the canopy in unprecedented spectral and spatial resolution. Fisher et al. [70] identified the modeling of the radiative transfer through the canopy as one of the biggest sources of uncertainty in current ecosystem and vegetation dynamics models. We suggest using 3D modeling approaches to exploit these complex interactions and evaluate the influence of simplifications in the radiative transfer on e.g. modeled absorbed photosynthetically active radiation (APAR), gross primary productivity (GPP), or light availability. The combination of experimental data and 3D modeling can reveal substantial insights to advance understanding of light-related mechanisms driving species coexistence, competition and diversity in complex forest canopies.

\section{APPENDIX A \\ UNCERTAINTY CONSIDERATIONS}

\section{A. ASD radiometric uncertainty}

The ASD radiometric uncertainty was based on an uncertainty budget provided by ASD. It is traceable to the NIST irradiance scale and includes further components (lamp current, distance from lamp to panel, panel reflectance, wavelength calibration and instrument long-term stability). The uncertainty is in the range to be expected from such calibration setups as used by ASD, e.g. the radiance transfer standard RASTA shows similar uncertainties [71].

\section{B. Spectralon panel uncertainty}

Spectralon panels suffer from a number of uncertainties. These comprise the reflective properties as well as short-term and long-term degradations. The reflectance of the panel is different from unity, and the reflectance factors of the panel and the associated uncertainties are defined in the calibration report given by the manufacturer [72]. These reflectance factors and uncertainties are however only true for the illumination and observation angles during calibration. Information about the specific bidirectional reflectance distribution function (BRDF) of Spectralon panels can be obtained by calibration in specialized laboratories [73]. Short term degradations involve the accumulation of particles during the field use and have been reported to range around $0.5 \%$ [73], but are obviously largely dependent on the environmental conditions and should be assessed specifically during each field experiment. Long term changes in reflectivity are not only associated with exposure to high levels of UV radiation, but also due to the storage [62]. The panels used during this experiment are not strictly traceable to a standard due to their storage and field usage since their last calibration. To account for the resulting uncertainties, we include the following uncertainty budget components: uncertainty of reflectance as provided by supplier and uncertainty due to storage and use. The latter was estimated from a comparative experiment, measuring the reflected solar irradiance over eight different panels during stable midday conditions in summer 2015. The storage and use uncertainty utilized in this study was calculated as the mean reflectance factor difference relative to the most recently calibrated panel.

\section{Angular uncertainty of the gimbal}

The uncertainty contribution to the total uncertainty associated with the irradiance measurements due to the leveling performance of the gimbal was assessed by measuring the angular displacement from a perfect horizontal alignment after a movement of the whole measurement device. This angular displacement was 


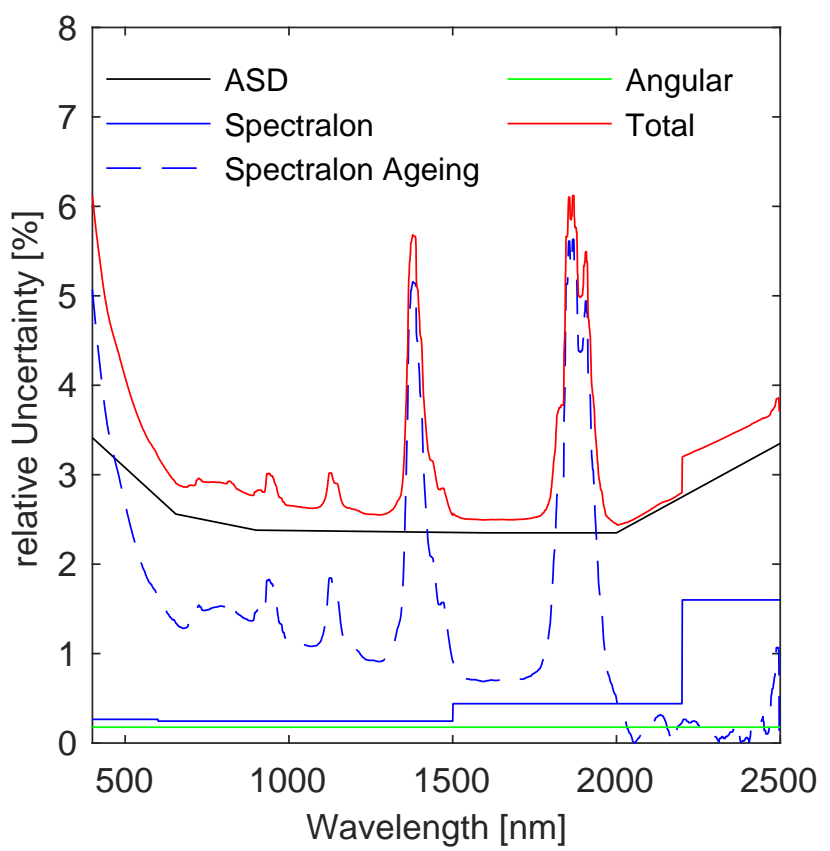

Fig. A1. Relative uncertainty contributions to the total uncertainty associated with the irradiance measurements using an ASD hand-held spectrometer and a spectralon panel mounted on a gimbal

measured 20 times to receive an average angular displacement ( 0.23 degrees) from the perfect horizontal alignment. The contribution of the angular displacement to the total uncertainty of the measured irradiance was performed by calculating the expected irradiance on a completely flat surface based on the measured irradiance of the tilted surface defined by the tilt angles and the solar angle as described in [74]. According to Gulin et al. [74], calculation of the direct irradiance incident on a tilted surface $B_{\varphi}$ is purely geometrical:

$$
B_{\varphi}=\frac{B_{h}}{\cos \theta_{z}} \cos \theta=B_{h} r_{b}
$$

where $B_{h}$ is the direct horizontal solar irradiance, $r_{b}$ is the direct irradiance conversion factor

$$
r_{b}=\max \left(0, \frac{\cos \theta}{\cos \theta_{z}}\right)
$$

and $\Theta$ is the angle of incidence, e.e., the angle between the sun direction and the normal direction of a tilted surface:

$$
\cos \theta=\cos \theta_{z} \cos \beta+\sin \theta_{z} \sin \beta \cos \left(\gamma_{s}-\gamma\right)
$$

where $\theta_{z}$ and $\gamma_{s}$ are the solar zenith and azimuth angles respectively. $\gamma$ and $\beta$ are the measured angular displacement of the spectralon panel from a perfect horizontal alignment. As we measured the irradiance on the tilted surface $\left(B_{\varphi}\right)$ we have to solve equation 7 for $B_{h}$. The difference between $B_{h}$ and $B_{\varphi}$ then gives the uncertainty associated with the irradiance measurement due to the alignment performance of the gimbal.

\section{Total uncertainty associated with irradiance measurements}

Fig. A1 shows the different contributions to the total relative uncertainty associated with the irradiance measurements. Note that the uncertainty associated with the spectralon panel does not account for aging effects as it is based on a calibration certificate of a new pristine spectralon panel. For older, used spectralon panels, the uncertainty contribution of the spectralon panel is assumed to be much larger especially in the visible spectral range. 


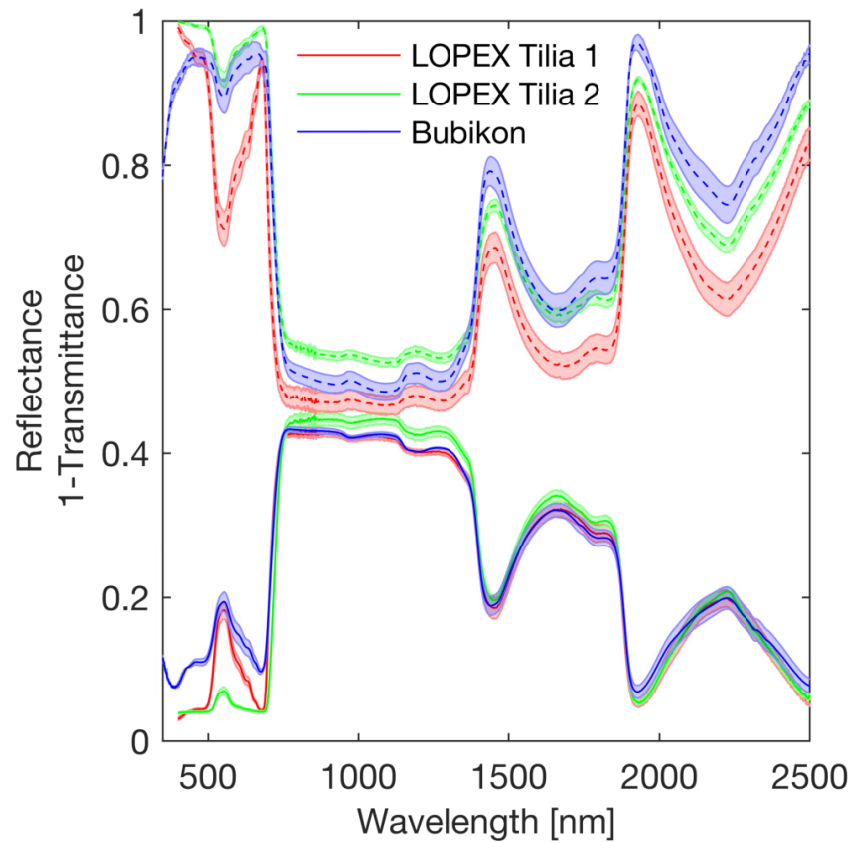

Fig. A2. Comparison between the two LOP entries for Tilia trees in the LOPEX database [75] and the measured LOP for this experiment (Bubikon).

\section{E. Influence of LOP on simulated irradiance}

Extracted leaf optical properties to paramiterize the DART model may be influenced by significant uncertainties. However, an estimation of the uncertainty associated with the retrieved LOP is a non-trivial task. In order to assess the impact of changing LOP on the modeled irradiance, we performed additional DART simulations, where we changed the LOP based on literature values found for the same tree species (tilia), but with a different genus (platyphyllos instead of cordata) in the LOPEX database [75]. Even though the LOP found in the LOPEX database show large variance in reflectance and transmittance (see Fig. A2), it can give us an idea about the range in LOP for tilia trees. We performed four additional DART simulations with the two LOP found in the LOPEX database for transect \#7 and \#19 at 540nm and at $781 \mathrm{~nm}$ (also compare with Fig. 10). We opted for a wavelength at 540nm instead of 470nm, as the reflectance and transmittance values show larger differences at this wavelength, possibly showing also larger impacts on modeled irradiance values. Fig. A3 shows the range and the difference in modeled irradiance when we use the LOPs derived from the LOPEX database as compared to the original simulation based on the measured LOP. Even though the difference in reflectance and transmittance of the measured leaves and retrieved form the LOPEX database is relatively large (up to $80 \%$ in transmittance at 540nm when measured transmittance is compared with transmittance for LOPEX Tilia 1), the impact on simulated irradiance is marginal. Outside shadowed areas, the difference in modeled irradiance is less than $0.2 \%$ at $540 \mathrm{~nm}$ with distance to tree trunk of more than $8 \mathrm{~m}$ and less than $1.7 \%$ at $781 \mathrm{~nm}$ after $6 \mathrm{~m}$ distance to tree trunk. Only within shadowed areas the relative difference between original simulation and the ones based on changed LOPs are larger (up to $22 \%$ at $540 \mathrm{~nm}$ and up to $12 \%$ at $781 \mathrm{~nm}$ ), however as the absolute amount of irradiance in these shadowed areas is very low, the absolute difference is nearly negligible with $0.004 \mathrm{~W} \mathrm{~m}^{-2} \mathrm{~nm}^{-1} \mathrm{sr}^{-1}$ and $0.009 \mathrm{~W} \mathrm{~m}^{-2} \mathrm{~nm}^{-1} \mathrm{sr}^{-1}$ at $540 \mathrm{~nm}$ and $781 \mathrm{~nm}$ respectively. We can therefore conclude that a change in LOP due to measurement errors would only have a minor effect on the modeled surface irradiance values.

\section{ACKNOWLEDGMENT}

The authors would like to thank Jan Pisek from the Tartu Observatory, Estonia for providing leaf angle measurements of a tilia cordata measured in Kew Gardens, London. They would also like to thank the 

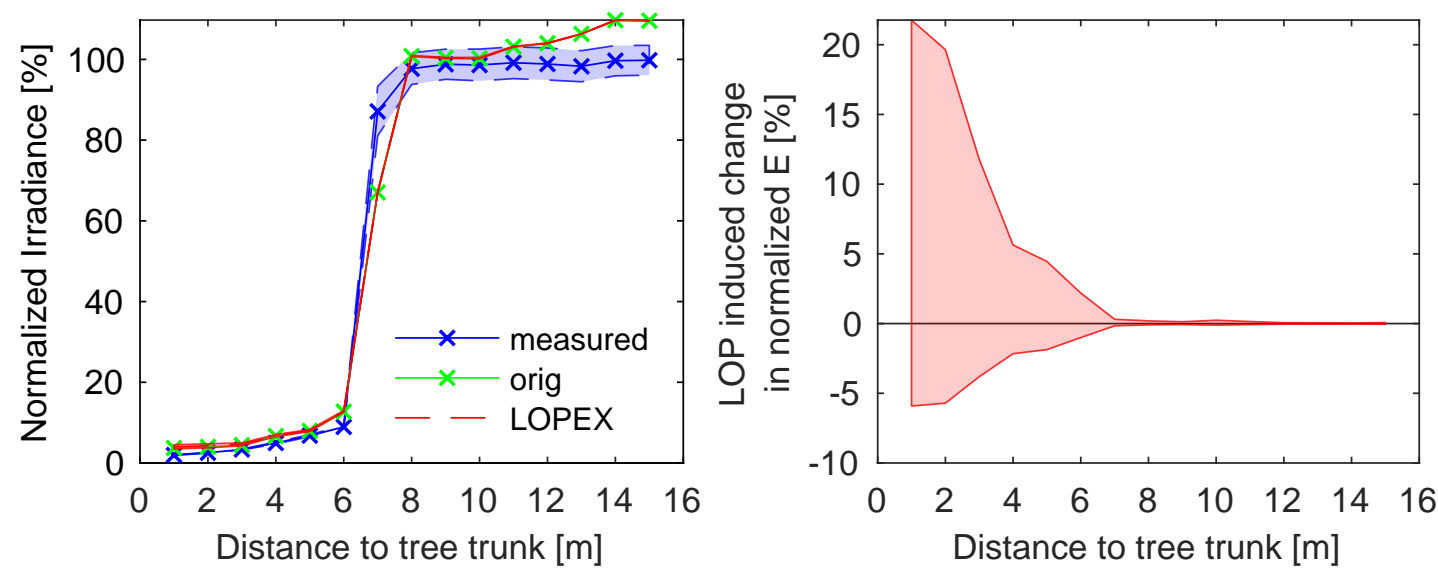

(a)
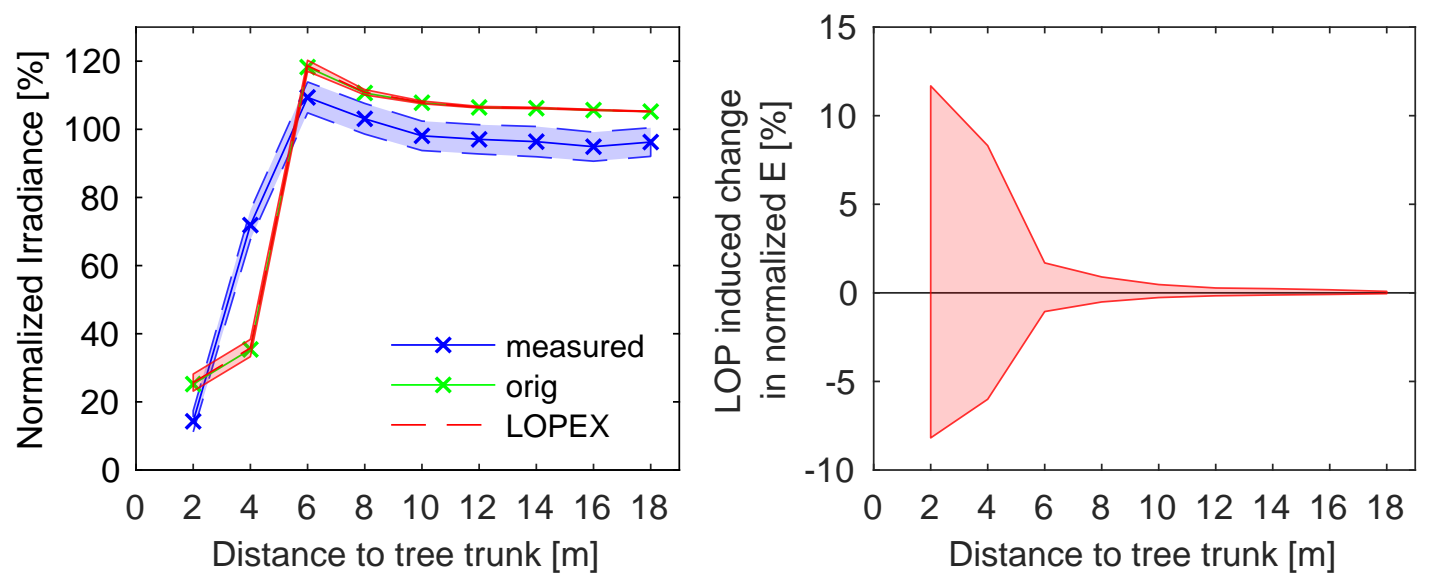

(b)

Fig. A3. Measured vs. simulated irradiance for transects \#7 (10a) at 540nm and \#19 (10b) at $781 \mathrm{~nm}$. Left panels show the normalized irradiance transects for measured (blue) and simulated irradiance with original LOP parameterization (green) and with LOP parameterization based on tilia entries found in the LOPEX database [75] in red. Irradiance normalization has been performed based on measured irradiance at the reference location. The red shaded area denotes the range in simulated irradiance based on the variance in LOP of the LOPEX databse. Right panels show relative change in normalized irradiance when comparing the original simulation with measured LOP with the simulations based on LOPEX LOP.

Science IT group S3IT (University of Zurich, UZH) and especially Sergio Maffioletti, for their support on running the Radiative transfer simulations on their cloud computing environment. We further acknowledge the help of Dr. Laura Mihai from the National Institute for Laser, Plasma and Radiation Physics, Romania, during her short term scientific mission at UZH. This work was supported by a STSM Grant from COST Action OPTIMISE ES1309. We would also like to thank the anonymous reviewers for their valuable comments.

\section{REFERENCES}

[1] M. E. Schaepman, S. L. Ustin, A. J. Plaza, T. H. Painter, J. Verrelst, and S. Liang, "Earth system science related imaging spectroscopy-An assessment," Remote Sensing of Environment, vol. 113, pp. S123-S137, 2009.

[2] M. E. Schaepman, M. Jehle, A. Hueni, P. D’Odorico, A. Damm, J. Weyermann, F. D. Schneider, V. Laurent, C. Popp, F. C. Seidel, K. Lenhard, P. Gege, C. Küchler, J. Brazile, P. Kohler, L. De Vos, K. Meuleman, R. Meynart, D. Schläpfer, M. Kneubühler, and K. I. Itten, "Advanced radiometry measurements and Earth science applications with the Airborne Prism Experiment (APEX)," Remote Sensing of Environment, vol. 158, pp. 207-219, mar 2015.

[3] S. L. Ustin, A. a. Gitelson, S. Jacquemoud, M. Schaepman, G. P. Asner, J. a. Gamon, and P. Zarco-Tejada, "Retrieval of foliar information about plant pigment systems from high resolution spectroscopy," Remote Sensing of Environment, vol. 113, pp. S67-S77, sep 2009. 
[4] F. D. Schneider, F. Morsdorf, B. Schmid, O. L. Petchey, A. Hueni, D. S. Schimel, and M. E. Schaepman, "Mapping functional diversity from remotely sensed morphological and physiological forest traits," Nature Communications, vol. 8, no. 1, p. 1441 , dec 2017.

[5] A. Damm, L. Guanter, E. Paul-Limoges, C. van der Tol, A. Hueni, N. Buchmann, W. Eugster, C. Ammann, and M. E. Schaepman, "Far-red sun-induced chlorophyll fluorescence shows ecosystem-specific relationships to gross primary production: An assessment based on observational and modeling approaches," Remote Sensing of Environment, vol. 166, pp. 91-105, 2015.

[6] A. Damm, L. Guanter, W. Verhoef, D. Schläpfer, S. Garbari, and M. Schaepman, "Impact of varying irradiance on vegetation indices and chlorophyll fluorescence derived from spectroscopy data," Remote Sensing of Environment, vol. 156, pp. 202-215, 2015.

[7] D. Fawcett, W. Verhoef, D. Schläpfer, F. Schneider, M. Schaepman, and A. Damm, "Advancing retrievals of surface reflectance and vegetation indices over forest ecosystems by combining imaging spectroscopy, digital object models, and 3D canopy modelling," Remote Sensing of Environment, vol. 204, pp. 583-595, jan 2018.

[8] R. Richter, "A fast atmospheric correction algorithm applied to Landsat TM images," International Journal of Remote Sensing, vol. 11, no. 1, pp. 159-166, 1990.

[9] R. Richter and A. Müller, "Deshadowing of satellite/airborne imagery," International Journal of Remote Sensing, vol. 26, no. 15, pp. 3137-3148, 2005.

[10] G. P. Asner, R. E. Martin, C. B. Anderson, and D. E. Knapp, "Quantifying forest canopy traits: Imaging spectroscopy versus field survey," Remote Sensing of Environment, vol. 158, pp. 15-27, 2015.

[11] Z. Malenovský, L. Homolová, R. Zurita-Milla, P. Lukě̌, V. Kaplan, J. Hanuš, J. P. Gastellu-Etchegorry, and M. E. Schaepman, "Retrieval of spruce leaf chlorophyll content from airborne image data using continuum removal and radiative transfer," Remote Sensing of Environment, vol. 131, pp. 85-102, 2013.

[12] S. M. Adler-Golden, M. W. Matthew, G. P. Anderson, G. W. Felde, and J. A. Gardner, "An Algorithm for De-Shadowing Spectral Imagery," in SPIE, vol. 4816, 2002, pp. 203-210.

[13] O. Friman, G. Tolt, and J. Ahlberg, "Illumination and shadow compensation of hyperspectral images using a digital surface model and non-linear least squares estimation," Proceedings of SPIE Remote Sensing 2011, no. August 2015, p. 8180, 2011.

[14] K. Adeline, M. Chen, X. Briottet, S. Pang, and N. Paparoditis, "Material reflectance retrieval in urban tree shadows with physics-based empirical atmospheric correction," in Urban Remote Sensing Event (JURSE), 2013 Joint, no. 2, Sao Paulo, 2013, pp. $279-283$.

[15] J. Gastellu-Etchegorry, E. Grau, and N. Lauret, "DART : A 3D Model for Remote Sensing Images and Radiative Budget of Earth Surfaces," in Modeling and Simulation in Engineering, C. Alexandru, Ed. Toulouse, France: CESBIO - CNES, CNRS (UMR 5126), IRD, Universite de Toulouse, 2012, pp. 1-40.

[16] J.-P. Gastellu-Etchegorry, T. Yin, N. Lauret, T. Cajgfinger, T. Gregoire, E. Grau, J.-B. Feret, M. Lopes, J. Guilleux, G. Dedieu, Z. Malenovský, B. Cook, D. Morton, J. Rubio, S. Durrieu, G. Cazanave, E. Martin, and T. Ristorcelli, "Discrete Anisotropic Radiative Transfer (DART 5) for Modeling Airborne and Satellite Spectroradiometer and LIDAR Acquisitions of Natural and Urban Landscapes," Remote Sensing, vol. 7, no. 2, pp. 1667-1701, 2015.

[17] A. R. Huete, "Soil and sun angle interactions on partial canopy spectra," International Journal of Remote Sensing, vol. 8, no. 9, pp. 1307-1317, 1987.

[18] D. A. Roberts, J. B. Adams, and M. O. Smith, "Predicted distribution of visible and near-infrared radiant flux above and below a transmittant leaf," Remote Sensing of Environment, vol. 34, no. 1, pp. 1-17, 1990.

[19] D. A. Roberts, "Separating Spectral Mixtures of Vegetation and Soils," Ph.D. dissertation, University of Washington, 1991.

[20] J. Stuckens, B. Somers, S. Delalieux, W. W. Verstraeten, and P. Coppin, "The impact of common assumptions on canopy radiative transfer simulations: A case study in Citrus orchards," Journal of Quantitative Spectroscopy and Radiative Transfer, vol. 110, no. 1-2, pp. 1-21, 2009.

[21] L. Tits, B. Somers, J. Stuckens, J. Farifteh, and P. Coppin, "Integration of in situ measured soil status and remotely sensed hyperspectral data to improve plant production system monitoring: Concept, perspectives and limitations," Remote Sensing of Environment, vol. 128, pp. 197-211, 2013.

[22] J. van Beek, L. Tits, B. Somers, T. Deckers, P. Janssens, and P. Coppin, "Reducing background effects in orchards through spectral vegetation index correction," International Journal of Applied Earth Observation and Geoinformation, vol. 34, no. 1, pp. 167-177, 2015.

[23] D. K. Lynch, "Shadows," Applied Optics, vol. 54, no. 4, p. B154, feb 2015.

[24] T. Hilker, N. C. Coops, F. G. Hall, T. A. Black, M. A. Wulder, Z. Nesic, and P. Krishnan, "Separating physiologically and directionally induced changes in PRI using BRDF models," Remote Sensing of Environment, vol. 112, no. 6, pp. 2777-2788, 2008.

[25] T. Hilker, N. C. Coops, C. R. Schwalm, R. S. Jassal, T. A. Black, and P. Krishnan, "Effects of mutual shading of tree crowns on prediction of photosynthetic light-use efficiency in a coastal Douglas-fir forest." Tree physiology, vol. 28, no. 6, pp. 825-834, 2008.

[26] T. L. Takala and M. Mõttus, "Spatial variation of canopy PRI with shadow fraction caused by leaf-level irradiation conditions," Remote Sensing of Environment, vol. 182, pp. 99-112, 2016.

[27] M. Mõttus, T. L. H. Takala, P. Stenberg, Y. Knyazikhin, B. Yang, and T. Nilson, "Diffuse sky radiation influences the relationship between canopy PRI and shadow fraction," ISPRS Journal of Photogrammetry and Remote Sensing, vol. 105, pp. 54-60, 2015.

[28] J. P. Gastellu-Etchegorry, E. Martin, and F. Gascon, "DART: A 3D model for simulating satellite images and studying surface radiation budget," International Journal of Remote Sensing, vol. 25, no. 1, pp. 73-96, 2004.

[29] J. P. Gastellu-Etchegorry, "3D modeling of satellite spectral images, radiation budget and energy budget of urban landscapes," Meteorology and Atmospheric Physics, vol. 102, no. 3-4, pp. 187-207, 2008.

[30] F. D. Schneider, R. Leiterer, F. Morsdorf, J.-P. Gastellu-Etchegorry, N. Lauret, N. Pfeifer, and M. E. Schaepman, "Simulating imaging spectrometer data: 3D forest modeling based on LiDAR and in situ data," Remote Sensing of Environment, vol. 152, pp. 235-250, sep 2014.

[31] A. Berk, L. Bernstein, and D. Robertson, "MODTRAN: A Moderate Resolution Model For LOWTRAN," Spectral Sciences. Inc., Burlington, Tech. Rep., 1987. 
[32] M. Abegg, D. Kükenbrink, J. Zell, M. E. Schaepman, and F. Morsdorf, "Terrestrial laser scanning for forest inventories-tree diameter distribution and scanner location impact on occlusion," Forests, vol. 8, no. 6, pp. 1-29, 2017.

[33] D. Kükenbrink, F. D. Schneider, R. Leiterer, M. E. Schaepman, and F. Morsdorf, "Quantification of hidden canopy volume of airborne laser scanning data using a voxel traversal algorithm," Remote Sensing of Environment, vol. 194, no. Special Issue of the SilviLaser 2015 Conference, pp. 424-436, jun 2017.

[34] G. Vincent, C. Antin, M. Laurans, J. Heurtebize, S. Durrieu, C. Lavalley, and J. Dauzat, "Mapping plant area index of tropical evergreen forest by airborne laser scanning. A cross-validation study using LAI2200 optical sensor," Remote Sensing of Environment, vol. 198, pp. 254-266, 2017.

[35] M. Kazhdan and H. Hoppe, "Screened poisson surface reconstruction," ACM Transactions on Graphics, vol. 32, no. 3, pp. 1-13, 2013.

[36] P. Cignoni, P. Cignoni, M. Callieri, M. Callieri, M. Corsini, M. Corsini, M. Dellepiane, M. Dellepiane, F. Ganovelli, F. Ganovelli, G. Ranzuglia, and G. Ranzuglia, "MeshLab: an Open-Source Mesh Processing Tool," Sixth Eurographics Italian Chapter Conference, pp. 129-136, 2008.

[37] J. R. Miller, M. D. Steven, and T. H. Demetriades-Shah, "Reflection of layered bean leaves over different soil backgrounds: measured and simulated spectra," International Journal of Remote Sensing, vol. 13, no. 17, pp. 3273-3286, nov 1992.

[38] R. D. Jackson, T. R. Clarke, and M. Susan Moran, "Bidirectional calibration results for 11 spectralon and 16 BaSO4reference reflectance panels," Remote Sensing of Environment, vol. 40, no. 3, pp. 231-239, 1992.

[39] A. Hueni, J. Nieke, J. Schopfer, M. Kneubühler, and K. I. Itten, "The spectral database SPECCHIO for improved long-term usability and data sharing," Computers and Geosciences, vol. 35, no. 3, pp. 557-565, 2009.

[40] T. H. Hemmer, T. L. Westphal, L. Jackson, and M. Hwy, "Lessons learned in the post-processing of field spectroradiometric data covering the 0. 4 to 2.5 im wavelength region," Proceedings of SPIE, vol. 4049, pp. 249-260, 2000.

[41] A. Hueni and A. Bialek, "Cause, Effect, and Correction of Field Spectroradiometer Interchannel Radiometric Steps," IEEE Journal of Selected Topics in Applied Earth Observations and Remote Sensing, vol. 10, no. 4, pp. 1542-1551, 2017.

[42] A. Hueni, A. Damm, M. Kneubuehler, D. Schlapfer, and M. E. Schaepman, "Field and Airborne Spectroscopy Cross Validation -Some Considerations," IEEE Journal of Selected Topics in Applied Earth Observations and Remote Sensing, vol. 10, no. 3, pp. 1117-1135, 2017.

[43] J. Gamon, J. Peñuelas, and C. Field, "A narrow-waveband spectral index that tracks diurnal changes in photosynthetic efficiency," pp. $35-44,1992$.

[44] J. A. Gamon, L. Serrano, and J. S. Surfus, "The photochemical reflectance index: An optical indicator of photosynthetic radiation use efficiency across species, functional types, and nutrient levels," Oecologia, vol. 112, no. 4, pp. 492-501, 1997.

[45] J. A. Gamon, O. Kovalchuck, C. Y. Wong, A. Harris, and S. R. Garrity, "Monitoring seasonal and diurnal changes in photosynthetic pigments with automated PRI and NDVI sensors," Biogeosciences, vol. 12, no. 13, pp. 4149-4159, 2015.

[46] A. A. Gitelson, G. P. Keydan, and M. N. Merzlyak, "Three-band model for noninvasive estimation of chlorophyll, carotenoids, and anthocyanin contents in higher plant leaves," Geophysical Research Letters, vol. 33, no. 11, pp. 2-6, 2006.

[47] M. Béland, J.-L. Widlowski, R. A. Fournier, J.-F. Côté, and M. M. Verstraete, "Estimating leaf area distribution in savanna trees from terrestrial LiDAR measurements," Agricultural and Forest Meteorology, vol. 151, no. 9, pp. 1252-1266, sep 2011.

[48] M. Béland, D. D. Baldocchi, J.-L. Widlowski, R. A. Fournier, and M. M. Verstraete, "On seeing the wood from the leaves and the role of voxel size in determining leaf area distribution of forests with terrestrial LiDAR," Agricultural and Forest Meteorology, vol. 184, pp. 82-97, jan 2014.

[49] M. Béland, J.-L. Widlowski, and R. A. Fournier, "A model for deriving voxel-level tree leaf area density estimates from ground-based LiDAR," Environmental Modelling \& Software, vol. 51, pp. 184-189, jan 2014.

[50] E. Grau, S. Durrieu, R. Fournier, J.-p. Gastellu-etchegorry, and T. Yin, "Estimation of 3D vegetation density with Terrestrial Laser Scanning data using voxels . A sensitivity analysis of influencing parameters ." Remote Sensing of Environment, vol. 191, pp. 373-388, 2017.

[51] J. B. Feret, C. François, G. P. Asner, A. A. Gitelson, R. E. Martin, L. P. Bidel, S. L. Ustin, G. le Maire, and S. Jacquemoud, "PROSPECT4 and 5: Advances in the leaf optical properties model separating photosynthetic pigments," Remote Sensing of Environment, vol. 112, no. 6, pp. 3030-3043, 2008.

[52] P. Lukeš, L. Homolová, M. Navrátil, and J. Hanuš, "Assessing the consistency of optical properties measured in four integrating spheres," International Journal of Remote Sensing, vol. 38, no. 13, pp. 3817-3830, 2017.

[53] A. Hovi, P. Forsström, M. Mõttus, and M. Rautiainen, "Evaluation of Accuracy and Practical Applicability of Methods for Measuring Leaf Reflectance and Transmittance Spectra," Remote Sensing, vol. 10, no. 2, p. 25, dec 2017.

[54] G. P. Asner, "Biophysical and Biochemical Sources of Variability in Canopy Reflectance," Remote Sensing of Environment, vol. 64, no. 3, pp. 234-253, jun 1998.

[55] M. Alonzo, B. Bookhagen, J. P. McFadden, A. Sun, and D. A. Roberts, "Mapping urban forest leaf area index with airborne lidar using penetration metrics and allometry," Remote Sensing of Environment, vol. 162, pp. 141-153, jun 2015.

[56] W. Verhoef, C. V. D. Tol, and E. M. Middleton, "Vegetation Canopy Fluorescence and Reflectance Retrieval By Model Inversion Using Optimization," 5th International Workshop on Remote Sensing of Vegetation Fluorescence, no. 1, pp. 759-770, 2014.

[57] J. J. Richardson, L. M. Moskal, and S.-H. Kim, "Modeling approaches to estimate effective leaf area index from aerial discrete-return LIDAR," Agricultural and Forest Meteorology, vol. 149, no. 6-7, pp. 1152-1160, jun 2009.

[58] C. Borel, K. Ewald, M. Manzardo, C. Wamsley, and J. Jacobson, "Adjoint Radiosity based Algorithms for retrieving target Reflectances in urban area shadows," in Proceedings of 6th EARSeL SIG IS workshop, Tel Aviv, 2009.

[59] M. Disney, P. Lewis, and P. Saich, "3D modelling of forest canopy structure for remote sensing simulations in the optical and microwave domains," Remote Sensing of Environment, vol. 100, no. 1, pp. 114-132, jan 2006.

[60] V. C. E. Laurent, "Coupled canopy-atmosphere modelling for radiance-based estimation of vegetation properties," Ph.D. dissertation, Wageningen University, 2013. 
[61] T. Yin, J. P. Gastellu-Etchegorry, N. Lauret, E. Grau, and J. Rubio, “A new approach of direction discretization and oversampling for 3D anisotropic radiative transfer modeling," Remote Sensing of Environment, vol. 135, pp. 213-223, 2013.

[62] W. Möller, K. P. Nikolaus, and A. Höpe, "Degradation of the diffuse reflectance of spectralon under low-level irradiation," Metrologia, vol. 40, no. 1 SPEC., 2003.

[63] N. Fox, J. Aiken, J. Barnett, X. Briottet, R. Carvell, C. Frohlich, S. Groom, O. Hagolle, J. Haigh, H. Kieffer, J. Lean, D. Pollock, T. Quinn, M. Sandford, M. Schaepman, K. Shine, W. Schmutz, P. Teillet, K. Thome, M. Verstraete, and E. Zalewski, "Traceable radiometry underpinning terrestrial- and helio-studies (TRUTHS)," Advances in Space Research, vol. 32, no. 11, pp. 2253-2261, dec 2003.

[64] G. P. Asner, "Carnegie Airborne Observatory: in-flight fusion of hyperspectral imaging and waveform light detection and ranging for three-dimensional studies of ecosystems," Journal of Applied Remote Sensing, vol. 1, no. 1, p. 013536, sep 2007.

[65] C. C. Borel and S. A. Gerstl, "Nonlinear spectral mixing models for vegetative and soil surfaces," Remote sensing of environment, vol. 47, no. 3, pp. 403-416, 1994.

[66] T. W. Ray and B. C. Murray, "Nonlinear spectral mixing in desert vegetation," Remote Sensing of Environment, vol. 55, no. 1, pp. 59-64, 1996.

[67] D. A. Roberts, M. O. Smith, and J. B. Adams, "Green,Vegetation, Nonphotosynthetic Vegetation, and Soil in AVIRIS Data," Remote Sensing of Environment, vol. V. 44, no. N. 2/3, pp. p. 255-269, 1993.

[68] B. Somers, K. Cools, S. Delalieux, J. Stuckens, D. Van der Zande, W. W. Verstraeten, and P. Coppin, "Nonlinear Hyperspectral Mixture Analysis for tree cover estimates in orchards," Remote Sensing of Environment, vol. 113, no. 6, pp. 1183-1193, 2009.

[69] B. Somers, L. Tits, and P. Coppin, "Quantifying nonlinear spectral mixing in vegetated areas: Computer simulation model validation and first results," IEEE Journal of Selected Topics in Applied Earth Observations and Remote Sensing, vol. 7, no. 6, pp. 1956-1965, 2014.

[70] R. A. Fisher, C. D. Koven, W. R. L. Anderegg, B. O. Christoffersen, M. C. Dietze, C. Farrior, J. A. Holm, G. Hurtt, R. G. Knox, P. J. Lawrence, J. W. Lichststein, M. Longo, A. M. Matheny, D. Medvigy, H. C. Muller-Landau, T. L. Powell, S. P. Serbin, H. Sato, J. Shuman, B. Smith, A. T. Trugman, T. Viskari, H. Verbeeck, E. Weng, C. Xu, X. Xu, T. Zhang, and P. Moorcroft, "Vegetation Demographics in Earth System Models: a review of progress and priorities," Global Change Biology, no. August, pp. 1-20, 2017.

[71] D. R. Taubert, J. Hollandt, P. Sperfeld, S. Pape, A. Höpe, K. O. Hauer, P. Gege, T. Schwarzmaier, K. Lenhard, and A. Baumgartner, "Providing radiometric traceability for the calibration home base of DLR by PTB," AIP Conference Proceedings, vol. 1531, pp. 376-379, 2013.

[72] D. Helder, K. Thome, D. Aaron, L. Leigh, J. Czapla-Myers, N. Leisso, S. Biggar, and N. Anderson, "Recent surface reflectance measurement campaigns with emphasis on best practices, SI traceability and uncertainty estimation," Metrologia, vol. 49, no. 2, 2012.

[73] A. J. Deadman, I. D. Behnert, N. P. Fox, and D. Griffith, "National Physical Laboratory ( NPL ), United Kingdom Council for Scientific and Industrial Research ( CSIR ), South Africa," in 2011 IEEE International Geoscience and Remote Sensing Symposium, 2011, pp. 3883-3886.

[74] M. Gulin, M. Vasak, and M. Baotic, "Estimation of the global solar irradiance on tilted surfaces," 17th International Conference on Electrical Drives and Power Electronics (EDPE 2013), no. i, p. 6, 2013.

[75] B. Hosgood, S. Jacquemoud, G. Andreoli, J. Verdebout, G. Pedrini, and G. Schmuck, "Leaf optical properties experiment 93 (LOPEX93)," Ispra Italy'European Commission, Joint Research Centre Institute of Remote Sensing Applications., 1995.

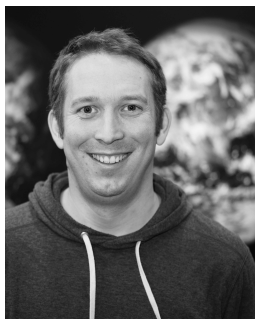

Daniel Kükenbrink received the B.Sc. and M.Sc. degree in geography from the University of Zurich (UZH), Zürich, Switzerland, in 2012 and 2014, respectively. He is currently a Ph.D. candidate at the Remote Sensing Laboratories, University of Zurich, Zurich, Switzerland. His research interests include remote sensing of vegetation structure and the radiative transfer through forest canopies.

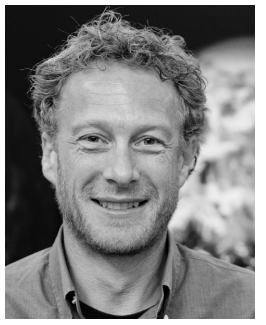

Andreas Hueni (M'12) received the B.Sc. degree in computer science from the University of Applied Science BruggWindisch, Brugg, Switzerland, in 1997, the PGDip degree in geographic information systems and M.Phil. (Sc.) degree in earth science from Massey University, Palmerston North, New Zealand, in 2005 and 2006, respectively, and the $\mathrm{Ph} . \mathrm{D}$. degree in geography from the University of Zurich (UZH), Zurich, Switzerland, in 2011. He has been working in the APEX Project since 2007 and is currently a Research Associate with the Remote Sensing Laboratories, UZH, responsible for APEX sensor and data calibration. His research interests include calibration of spectrometers and the design of combined database and software systems such as the spectral database SPECCHIO. 


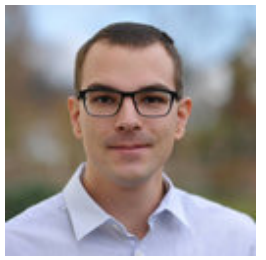

Fabian D. Schneider received the M.Sc and Ph.D. degrees in geography from the University of Zurich (UZH), Zürich, Switzerland in 2013 and 2018 respectively. He is currently a postdoc in the Carbon Cycle and Ecosystems group at the NASA Jet Propulsion Laboratory, California Institute of Technology, Pasadena, USA. His research interests includes the characterization of vegetation through the measurement of plant functional traits and trait diversity to study the impact of global change on plant health as well as ecosystem productivity and stability. His main focus lies on combining imaging spectroscopy and laser scanning measurements at different scales, and integrating it with physically based models.

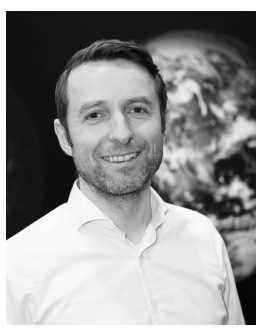

Alexander Damm received the Diploma (M.Sc.) degree and the Ph.D. (Dr.rer.Nat.) degree in geography from the Humboldt University, Berlin, Germany, in 2004 and 2008, respectively. From 2008 to 2014 he was a Postdoctoral researcher and from 2014 to 2017 group leader and project manager of the Swiss Earth Observatory Network at the Remote Sensing Laboratories, University of Zurich, Switzerland, respectively. In 2016, he became member of the Mission Advisory Group for European Space Agency's upcoming Fluorescence Explorer (FLEX) mission. In 2017, he was appointed as Assistant Professor Tenure Track for Remote Sensing of Water Systems at the University of Zurich. His research interests include the development of approaches based upon novel Earth Observation (EO) data and physical models to study biosphere-atmosphere interactions, ecosystem processes, as well carbon and water cycle dynamics.

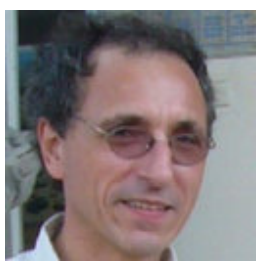

Jean-Philippe Gastellu-Etchegorry received the B.Sc. degree in electricity from ENSEEIHT, Toulouse, France, in 1978, the Aggregation degree in physics from Paris VI University, Paris, France, in 1981, and the Ph.D. degrees in solar physics in 1983, and remote sensing in 1989, from Paul Sabatier University (UPS), Toulouse, France. From 1984 to 1990, he worked in PUSPICSRemote Sensing Center, Gadjah Mada University, Yogyakarta, Indonesia, and SEAMEO, Bangkok, Thailand. Then, he joined the Centre dÉtude Spatiale des Rayonnements, Toulouse, France. In 1995, he joined the Centre dÉtudes Spatiale de la Biosphère, Toulouse, France, where he is currently the Deputy Director. He is a Full-Time Professor in UPS. Since 1993, he has been conducting research on three-dimensional radiative transfer modeling and develops the discrete anisotropic radiative transfer model.

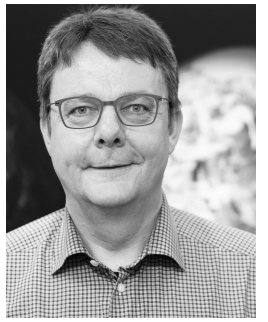

Michael E. Schaepman (M'05-SM'07) received the M.Sc. and Ph.D. degrees in geography from the University of Zurich (UZH), Zürich, Switzerland, in 1993 and 1998, respectively. In 1999, he was a Postdoctoral Researcher with the Optical Sciences Center, The University of Arizona, Tucson, AZ, USA. In 2000, he was appointed as a Project Manager of an APEX Spectrometer with the European Space Agency. In 2003, he became the Full Chair of geoinformation science and remote sensing with Wageningen University, Wageningen, The Netherlands. In 2009, he was appointed as the Full Chair of remote sensing with UZH, where he is currently the Head of the Remote Sensing Laboratories, Department of Geography. He is the Director of the University Research Priority ProgramGlobal Change and Biodiversity, and the Vice President for Veterinary Medicine and Science. His research interests include computational earth sciences using remote sensing and physical models, with a particular focus on the landatmosphere interface using imaging spectroscopy.

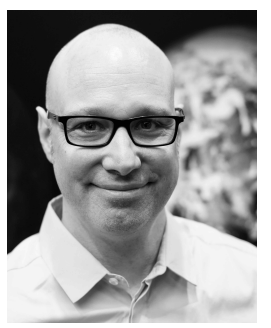

Felix Morsdorf received the M.Sc. degree in physical oceanography from the University of Kiel, Kiel, Germany, in 2001 and the Ph.D. degree in geography, with a specialization in LIDAR remote sensing, from the University of Zürich, Zürich, Switzerland, in 2007. He is a group leader with the Department of Geography, University of Zürich. His group uses empirical and physical approaches to bridge the semantic gap between data and information. His research is focused on laser scanning and vegetation structure. 